\title{
STABILITY ANALYSIS OF A SIGNALING CIRCUIT WITH DUAL SPECIES OF GTPASE SWITCHES
}

\author{
LUCAS M. STOLERMAN ${ }^{1}$, PRADIPTA GHOSH ${ }^{2,3,4 *}$, AND PADMINI RANGAMANI ${ }^{1 *}$
}

ABSTRACT. GTPases are molecular switches that regulate a wide range of cellular processes, such as organelle biogenesis, position, shape, and function, vesicular transport between organelles, and signal transduction. These hydrolase enzymes operate by toggling between an active "ON") guanosine triphosphate (GTP)-bound state and an inactive ("OFF") guanosine diphosphate (GDP)-bound state; such a toggle is regulated by GEFs (guanine nucleotide exchange factors) and GAPs (GTPase activating proteins). Here we propose a model for a network motif between monomeric $(\mathrm{m})$ and trimeric (t) GTPases assembled exclusively in eukaryotic cells of multicellular organisms. We develop a system of ordinary differential equations in which these two classes of GTPases are interlinked conditional to their ON/OFF states within a motif through coupling and feedback loops. We provide explicit formulae for the steady states of the system and perform classical local stability analysis to systematically investigate the role of the different connections between the GTPase switches. Interestingly, a coupling of the active mGTPase to the GEF of the tGTPase was sufficient to provide two locally stable states: one where both active/inactive forms of the mGTPase can be interpreted as having low concentrations and the other where both $\mathrm{m}$ - and tGTPase have high concentrations. Moreover, when a feedback loop from the GEF of the tGTPase to the GAP of the mGTPase was added to the coupled system, two other locally stable states emerged, both having the tGTPase inactivated and being interpreted as having low active tGTPase concentrations. Finally, the addition of a second feedback loop, from the active tGTPase to the GAP of the mGTPase, gives rise to a family of steady states that can be parametrized by a range of inactive tGTPase concentrations. Our findings reveal that the coupling of these two different GTPase motifs can dramatically change their steady state behaviors and shed light on how such coupling may impact signaling mechanisms in eukaryotic cells.

Date: December 15, 2020.

Key words and phrases. GTPases, Biochemical switches, Network motifs, Stability analysis.

${ }^{1}$ Department of Mechanical and Aerospace Engineering, University of California, San Diego, La Jolla CA 92093.

${ }^{2}$ Department of Medicine,University of California, San Diego, La Jolla, CA 92093.

${ }^{3}$ Department of Cellular and Molecular Medicine, University of California, San Diego, La Jolla, CA 92093.

${ }^{4}$ Moores Comprehensive Cancer Center, University of California, San Diego, La Jolla, CA 92093.

*To whom correspondence should be addressed. e-mail: prangamani@ucsd.edu; prghosh@health.ucsd.edu. 
1. Introduction $\quad 3$

2. Model Development $\quad 5$

2.1. Assumptions. $\quad 5$

2.2. Governing Equations. $r$

2.3. Nondimensionalization $r$

3. Mathematical analysis and results $\quad 8$

3.1. Forward coupling connection: Recruitment of tGEF by active mGTPases $\left(m G^{*} \rightarrow t G E F\right)$. 9

3.2. Coupled switches with feedback loop $t G E F \rightarrow m G A P:$ Recruitment of tGEF by active mGTPases and tGEF colocalization with mGAP

3.3. Coupled switches with feedback loops $t G E F \rightarrow m G A P$ and $t G^{*} \rightarrow m G A P$ : Recruitment of tGEF by active mGTPases, tGEF colocalization with mGAP, and activation of mGAP by active tGTPases

3.4. Numerical Simulations.

4. Discussion

5. Acknowledgments

References

Appendix A. Proof of Proposition 3.1

Steady states.

Local Stability Analysis.

Appendix C. Proof of Theorem 3.3 


\section{INTRODUCTION}

Each eukaryotic cell has many a large number of GTP-binding proteins (also called GTPases or G-proteins). They are thought to be intermediates in an extended cellular signaling and transport network that touches on nearly every aspect of cell function $[1,2,3]$. One unique feature of GTPases is that they serve as biochemical switches that exist in an 'OFF' state when bound to a guanosine diphosphate (GDP), and can be turned 'ON' when that GDP is exchanged for a guanosine triphosphate (GTP) nucleotide $[1,4]$. Turning the GTPase 'ON' is the key rate limiting step in the activation-inactivation process, requires an external stimulus, and is catalyzed by a class of enzymes called guanine nucleotide exchange factors (GEFs) [5]. G proteins return to their 'OFF' state when the bound GTP is hydrolyzed to guanosine diphosphate (GDP) via an intrinsic hydrolase activity of the GTPase; this step is catalyzed by GTPase-activating proteins (GAPs) [6]. Thus, GEFs and GAPs play a crucial role in controlling the dynamics of the GTPase switch and the finiteness of signaling that it transduces [7, 8, 9, 10]. Dysregulation of GTPase switches has been implicated in cellular malfunctioning and is commonly encountered in diverse diseases $[11,12,13,14]$. For example, hyperactivation of GTPases $[15,16]$ is known to support a myriad of cellular phenotypes that contribute to aggressive tumor traits $[17,18]$. Such traits have also been associated with aberrant activity of GAPs [15] or GEFs [19, 20, 21, 22, 23, 24]. These works underscore the importance of GTPases as vital regulators of high fidelity cellular communication.

There are two distinct types of GTPases that gate signals: small or monomeric $(\mathrm{m})$ and trimeric (t) GTPases. mGTPases are mostly believed to function within the cell's interior and are primarily concerned with organelle function and cytoskeletal remodeling $[25,26,27]$. tGTPases, on the other hand, were believed to primarily function at the cell's surface from where they gate the duration, type and extent of signals that are initiated by receptors on the cell's surface [28, 29]. These two classes of switches were believed to function largely independently, until early 1990's when tGTPases were detected on intracellular membranes, e.g., the Golgi [29, 30], and studies alluded to the possibility that they, alongside mGTPases, may co-regulate organelle function and structure [31]. But it was not until 2016 that the first evidence of an example of functional coupling between the two switches - $\mathrm{m}$ - and tGTPases- emerged. Using a combination of biochemical, biophysical, structural modeling, live cell imaging, and numerous readouts of Golgi functions, it was shown that $\mathrm{m}$ - and tGTPase co-regulate each other on the Golgi [32]. The specific discovery of GIV/Girdin, a non-receptor GEF for G $\alpha i$, as a platform for crosstalk between trimeric G proteins and monomeric Arf1 GTPases at the Golgi is the main biological motivation of the present study. In Fig 1A, we depict where these proteins interact in the cell and what experimentally-determined sequence of events, segregated in space and time, enable the execution of key steps in secretion through the Golgi. The experiments in [32] showed that when mGTPase (Arf family) 
is turned 'ON', it engages with a GEF for tGPTase (GIV/Girdin; tGEF); the latter binds and activates tGTPase, of the Gi subfamily, Goi. The engaged tGEF triggers the activation of a tGTPase (Gi). Upon activation, the tGTPases activate the GAP for Arf1, ArfGAP2/3 (mGAP), via the release of 'free' G $\beta \gamma$. The mGAP turns 'OFF' the mGTPase Arf1, thereby terminating the mGTPase signaling. Termination of the mGTPase (Arf1) activity results in a finite lifetime of the Arf1 signal. This "finiteness" of signal from Arf1 is critical for membrane trafficking and organelle structure [33, 34, 35]. Thus, this phenomenon of co-regulation between the two classes of GTPases was shown to be critical in limiting the duration of mGTPase and tGTPase signaling on the Golgi membrane, which in turn significantly regulates Golgi shape and function. In doing so, this dual GTPase circuit converted simple chemical signals into complex mechanical outputs such as membrane trafficking. Emerging evidence from protein-protein interaction networks and decades of work on both species of GTPases suggest that such co-regulation through coupling between the GTPases is possible and likely occurs on multiple organellar membranes. What advantages do two coupled species of GTPase switches provide over independent, uncoupled switches? The answer to this question has not yet been experimentally dissected or intuitively theorized.

Mathematical models of signaling networks have contributed significantly to our understanding of how network motifs might function [36, 37, 38, 39, 40, 41]. Continuous-time dynamical systems, commonly represented by systems of ordinary differential equations (ODEs), are powerful tools for building rich and insightful mathematical models [42]. For example, a comprehensive steady state analysis of ODE system helped frame the concept of "zero-order ultrasensitivity" where large responses in the active fraction of a protein of interest are driven by small changes in the saturated ratio of the enzymes [43]. Similarly, modeling biochemical networks with dynamical systems also has revealed the existence of bistable switches and biological oscillators within a feedback network architecture $[44,45,46]$. The simple system proposed by Ferrell and Xiong [46] served as a basis for modeling cellular all-or-none responses, and hence, crucial for decision-making within several signaling processes. Dynamical systems have also be used for mapping chemical reactions into differential equations; when numerically integrated (clustered) these systems can be used to predict the evolution of large reaction networks [47, 48, 49]. For example, clustering methods have revealed the existence of recurrent structures, the so-called "network motifs', the dynamics of which have been inferred with a Boolean kinetic system of differential equations [49]. These models produced various dynamic features corresponding to the motif structure, which allowed the understanding of underlying biology (i.e., gene expression patterns). Furthermore, studies using network ODE models have revealed that information is processed in cells through intricate connections between signaling pathways rather than individual motifs [50, 51]. From a systems biology modeling perspective, large systems of differential equations are usually hard to 
analyze, but when combined with experiments, they can give rise to quantitative predictions $[52,53,54,55,56,57,58,59,60]$.

Here we built a mathematical model to investigate the stability properties of the coupled $\mathrm{m}$ - and tGTPase switches, the first example of its kind, that has been observed experimentally [32]. Beginning with the uncoupled GTPase switches (Fig.1B) as our starting point, we specifically sought to understand the stability features of the coupled motif (Fig.1C). We proposed a system of ODEs and obtained the steady states of this new network motif to understand the input-output relationships. Given the model formulation and the fact that we do not know the various kinetic parameters, obtaining these states is critical to our understanding of this network behavior. Then we studied the dynamic behavior under small perturbation around these steady states using local stability analysis [61, 62]. We investigated the different coupling and feedback loops between these two motifs all representing the observed biochemical and biophysical events during signal transduction (Fig.1D). In Fig.1E, we summarize the steady-states of the system. Our analyses revealed the existence of steady-states and their stability depends on the network connectivity. In particular, the coupling between the two switches through the connection between $\mathrm{mG}^{*}$ and tGEF (represented by "1") allowed for the emergence of two steady states with low/high $\mathrm{mG}$ and $\mathrm{mG}^{*}$ concentrations, while $\mathrm{tG}^{*}$ steady state concentration remained high in both cases. On the other hand, low $\mathrm{tG}^{*}$ steady state concentrations were obtained when the feedback loop $t G E F \rightarrow m G A P$ was added to the system (represented by " $1+2$ "). Finally, the feedback loop $t G^{*} \rightarrow m G A P$ allowed for the emergence of four parametrized families of steady state within the same low/high configurations. In what follows, we present the model assumptions and derivation in $\S$ Section 2 , the local stability analysis and numerical simulations in §Section 3, and discuss our findings in the context of GTPase signalling networks in §Section 4.

\section{Model Development}

In this section, we introduce our mathematical model for the GTPase coupled circuit (Fig.1C). We begin with outlining the model assumptions in §Section 2.1 and describe the reactions and governing equations in detail in $\S$ Section 2.2.

2.1. Assumptions. Our model describes the time evolution of the concentrations for the different system components. Table 1 contains the set of reactions in our system. We explicitly allow for three connections between the $\mathrm{m}$ - and the $\mathrm{t}$-GTPase switches based on experimental findings [32] as described below.

- Arrow 1: Represents the coupling of the two switches and represents the recruitment/engagement of tGEF by $\mathrm{mG}^{*}$ (Figure 1A,B).

- Arrow 2: Represents the feedback from tGEF to mGAP (Figure 1A,B). 
(A)

(B)

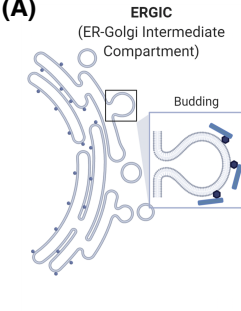

(C)

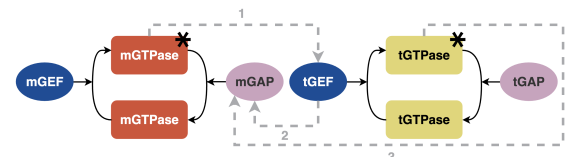

(D)

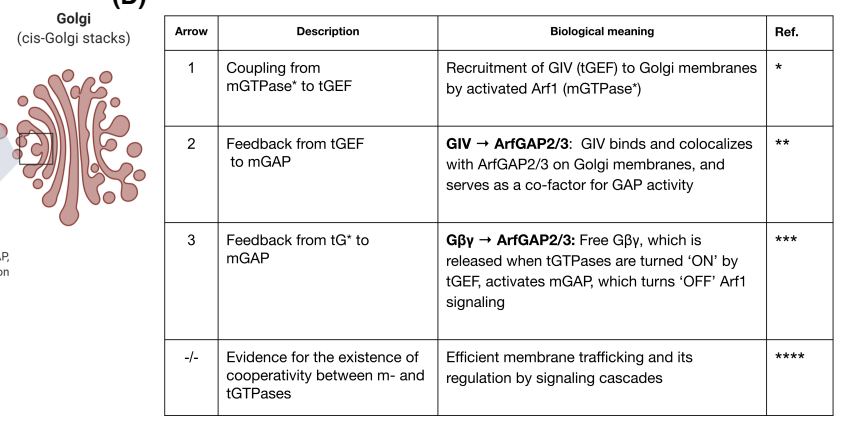

(E)

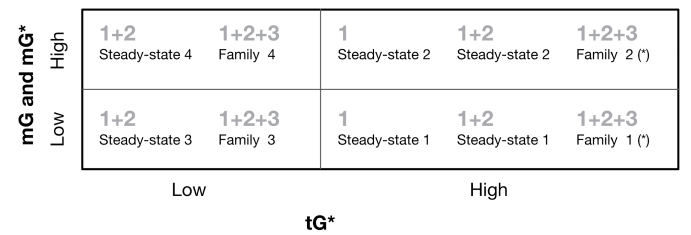

FIGURE 1. A network motif in which two species of GTPases are inter-

linked. (A) Recent experimental findings revealed that monomeric Arf GTPases and trimeric $G$ proteins co-regulate each other on the Golgi membrane (B) Uncoupled monomeric and trimeric GTPase switches are represented by mGTPase and tGTPase, respectively. The black star denotes the active forms. Activation and inactivation are regulated by GEFs and GAPs, where the first letter ( $\mathrm{m}$ or t) indicates the associated GTPase. (C) Our proposed mathematical model describes the interaction between the two GTPase switches. Arrows 1, 2, and 3 show the coupling and feedback loops that were found experimentally. (D) Description and biological meaning of each arrow connecting the GTPase switches. References: [32] for arrow $1(*),[32]$ for arrow $2(* *)$, [32, 63] for arrow $3(* * *)$, and $[63,30,64,65,31]$ for evidence of cooperativity between $m$ and tGTPases $(* * * *)$. (E) For the three combinations of arrows $(1,1+2$, and $1+2+3)$ chosen in our study, we calculate the steady state solutions for the coupled GTPase circuit model.

- Arrow 3: Represents the feedback from tG* to mGAP (Figure 1A,B).

Additionally, we only consider the toggling of GTPases that are mediated by GEFs and GAPs that activate and inactivate them, respectively.

To develop the model equations, we considered a well-mixed regime and that the concentrations of the species are in large enough amounts that deterministic kinetics hold $[66,67]$. Finally, for mathematical tractability, all reactions in the system are modeled using mass-action kinetics and nonlinear kinetics such as Hill functions or MichaelisMenten are not considered [68]. The reactions in the coupled circuit and the corresponding reaction rates used in the model are shown in Table 1.

2.2. Governing Equations. We developed a system of ODEs that describe coupled toggling of two switches, i.e., cyclical activation and inactivation of monomeric and trimeric 
TABLE 1. GTPase circuit reactions and rates used in the model

\begin{tabular}{cll}
\hline & List of Reactions & Reaction Rate \\
\hline $\mathrm{mG}^{*}$ activation & $\mathrm{mG}+\mathrm{mGEF}^{*} \stackrel{k_{o n}^{m G}}{\longrightarrow} \mathrm{mG}^{*}$ & $k_{o n}^{m G}\left[m G E F^{*}\right][m G]$ \\
$\mathrm{mG}^{*}$ inactivation & $\mathrm{mG}^{*}+\mathrm{mGAP}^{*} \stackrel{k_{o f f}^{m G}}{\longrightarrow} \mathrm{mG}$ & $k_{o f f}^{m G}\left[m G A P^{*}\right]\left[m G^{*}\right]$ \\
Coupling from $\mathrm{mG}^{*}$ to tGEF (arrow 1) & $\mathrm{mG}^{*}+\mathrm{tGEF} \stackrel{k_{o n}^{I}}{\longrightarrow} \mathrm{tGEF}^{*}$ & $k_{o n}^{I}[t G E F]\left[m G^{*}\right]$ \\
& & \\
$\mathrm{tG}^{*}$ activation & $\mathrm{tG}+\mathrm{tGEF}^{*} \stackrel{k_{o n}^{t G}}{\longrightarrow} \mathrm{tG}^{*}$ & $k_{o n}^{t G}\left[t G E F^{*}\right][t G]$ \\
$\mathrm{tG}^{*}$ inactivation & $\mathrm{tG}^{*}+\mathrm{tGAP}^{*} \stackrel{k_{o f f}^{t G}}{\longrightarrow} \mathrm{tG}$ & $k_{o f f}^{t G}\left[t G A P^{*}\right]\left[t G^{*}\right]$
\end{tabular}

Feedback loop from tGEF to mGAP (arrow 2) $\mathrm{tGEF}^{*}+\operatorname{mGAP} \stackrel{k_{o n}^{I I}}{\longrightarrow} \operatorname{mGAP}^{*} \quad k_{o n}^{I I}\left[t G E F^{*}\right][m G A P]$

Feedback loop $\mathrm{tG}^{*}$ to mGAP (arrow 3$) \quad \mathrm{tG}^{*}+\operatorname{mGAP} \stackrel{k_{o n}^{I I I}}{\longrightarrow} \mathrm{mGAP}^{*} \quad k_{o n}^{I I I}[m G A P]\left[t G^{*}\right]$

GTPases within the network motif shown in Fig.1C and described in Fig.1D. In what follows, the brackets represent concentrations, which are nonnegative real numbers. The system of equations are given by

$$
\begin{aligned}
\frac{d[m G]}{d t} & =-k_{o n}^{m G}\left[m G E F^{*}\right][m G]+k_{o f f}^{m G}\left[m G A P^{*}\right]\left[m G^{*}\right] \\
\frac{d\left[m G^{*}\right]}{d t} & =k_{o n}^{m G}\left[m G E F^{*}\right][m G]-k_{o f f}^{m G}\left[m G A P^{*}\right]\left[m G^{*}\right]-k_{o n}^{I}[t G E F]\left[m G^{*}\right] \\
\frac{d[t G]}{d t} & =-k_{o n}^{t G}\left[t G E F^{*}\right][t G]+k_{o f f}^{t G}\left[t G A P^{*}\right]\left[t G^{*}\right] \\
\frac{d\left[t G^{*}\right]}{d t} & =k_{o n}^{t G}\left[t G E F^{*}\right][t G]-k_{o f f}^{t G}\left[t G A P^{*}\right]\left[t G^{*}\right]-k_{o n}^{I I I}[m G A P]\left[t G^{*}\right] \\
\frac{d[t G E F]}{d t} & =-k_{o n}^{I}[t G E F]\left[m G^{*}\right] \\
\frac{d\left[t G E F^{*}\right]}{d t} & =k_{o n}^{I}[t G E F]\left[m G^{*}\right]-k_{o n}^{I I}\left[t G E F^{*}\right][m G A P] \\
\frac{d[m G A P]}{d t} & =-k_{o n}^{I I I}[m G A P]\left[t G^{*}\right]-k_{o n}^{I I}\left[t G E F^{*}\right][m G A P] \\
\frac{d\left[m G A P^{*}\right]}{d t} & =k_{o n}^{I I I}[m G A P]\left[t G^{*}\right]+k_{o n}^{I I}[m G A P]\left[t G E F^{*}\right]
\end{aligned}
$$

where the $k$ 's represent the reaction rate parameter for each reaction rate. Since all of reactions rates are second order, the $k$ 's have units of $1 /[\mu M \cdot s]$

To complete the system definition, all model components must have nonnegative initial conditions. We also assume that the concentrations of $\left[m G E F^{*}\right]$ and $\left[t G A P^{*}\right]$ are constant and nonzero in our model. In particular, if $k_{o n}^{I}=k_{o n}^{I I}=k_{o n}^{I I I}=0$, then our system describes two uncoupled GTPase switches (Fig.1B) such that each has the same dynamics of the single GTPase model proposed in [4]. 
2.3. Nondimensionalization. We introduce a nondimensional version of Eqs. 2.1 - 2.8 to reduce the number of free parameters and to obtain a new system of equations that is independent of the units of measurement. We denote $T=\left(k_{o n}^{m G}\left[m G E F^{*}\right]\right)^{-1}$ as be the characteristic time scale, $([T]=s)$. While there are many choices of time scales, this is the natural choice because it reflects the time scale of the coupling of the m- and t-GTPase switches. We define the characteristic concentration, $U_{\zeta}=\left[m G E F^{*}\right]$ for $\zeta \in$ $\left\{m G, m G^{*}, t G, t G^{*}, t G E F, t G E F^{*}, m G A P, m G A P^{*}\right\}$, with units $\left(\left[U_{\zeta}\right]=\mu M\right)$.

These characteristic quantities allow us to express the dimensionless kinetic rates as ratios between their dimensional forms and the rate of mGTPase activation $k_{o n}^{m G}$. In fact, defining

$$
\begin{gathered}
\widetilde{\rho_{o f f}^{m G}}=\frac{k_{o f f}^{m G}}{k_{o n}^{m G}}, \quad \widetilde{\rho_{o n}^{t G}}=\frac{k_{o n}^{t G}}{k_{o n}^{m G}}, \quad \widetilde{\rho_{o f f}^{t G}}=\frac{k_{o f f}^{t G}\left[t G A P^{*}\right]}{k_{o n}^{m G}\left[m G E F^{*}\right]}, \\
\widetilde{\rho_{o n}^{I}}=\frac{k_{o n}^{I}}{k_{o n}^{m G}}, \quad \widetilde{\rho_{o n}^{I I}}=\frac{k_{o n}^{I I}}{k_{o n}^{m G}}, \quad \widetilde{\rho_{o n}^{I I}}=\frac{k_{o n}^{I I I}}{k_{o n}^{m G}}, \quad \widetilde{t}=\frac{t}{k_{o n}^{m G}\left[m G E F^{*}\right]},
\end{gathered}
$$

and $\widetilde{[\zeta]}=\frac{[\zeta]}{\left[m G E F^{*}\right]}$ for $\zeta \in\left\{m G, m G^{*}, t G, t G^{*}, t G E F, t G E F^{*}, m G A P, m G A P^{*}\right\}$, we drop the tildes and write the system of dimensionless equations in the following form:

$$
\begin{aligned}
\frac{d[m G]}{d t} & =-[m G]+\rho_{o f f}^{m G}\left[m G A P^{*}\right]\left[m G^{*}\right] \\
\frac{d\left[m G^{*}\right]}{d t} & =[m G]-\rho_{o f f}^{m G}\left[m G A P^{*}\right]\left[m G^{*}\right]-\rho_{\text {on }}^{I}[t G E F]\left[m G^{*}\right] \\
\frac{d[t G]}{d t} & =-\rho_{\text {on }}^{t G}\left[t G E F^{*}\right][t G]+\rho_{o f f}^{t G}\left[t G^{*}\right] \\
\frac{d\left[t G^{*}\right]}{d t} & =\rho_{\text {on }}^{t G}\left[t G E F^{*}\right][t G]-\rho_{o f f}^{t G}\left[t G^{*}\right]-\rho_{\text {on }}^{I I I}[m G A P]\left[t G^{*}\right] \\
\frac{d[t G E F]}{d t} & =-\rho_{\text {on }}^{I}[t G E F]\left[m G^{*}\right] \\
\frac{d\left[t G E F^{*}\right]}{d t} & =\rho_{\text {on }}^{I}[t G E F]\left[m G^{*}\right]-\rho_{\text {on }}^{I I}\left[t G E F^{*}\right][m G A P] \\
\frac{d[m G A P]}{d t} & =-\rho_{\text {on }}^{I I I}[m G A P]\left[t G^{*}\right]-\rho_{\text {on }}^{I I}\left[t G E F^{*}\right][m G A P] \\
\frac{d\left[m G A P^{*}\right]}{d t} & =\rho_{\text {on }}^{I I I}[m G A P]\left[t G^{*}\right]+\rho_{\text {on }}^{I I}[m G A P]\left[t G E F^{*}\right]
\end{aligned}
$$

In section 3, we perform a local stability analysis of the system given by Eqs.2.9 - 2.16.

\section{MAThematical ANALYSis AND RESUlts}

In this section, we explore the role of the coupling of the two switches and feedback loops on the system dynamics. We refer to the nondimensional concentrations and rates of the system given by Eqs.2.9 - 2.16 as solely by concentrations and rates. First, it is 
convenient to rewrite our nondimensional ODE system in the form

$$
\frac{d \mathbf{x}}{d t}=S . \mathbf{v}(\mathbf{x})
$$

where $\mathbf{x}$ represents the vector of concentrations for the different components, $S$ is the stoichiometric matrix and $\mathbf{v}(\mathbf{x})$ is a vector with the different reaction rates $[69,70]$. Thus we define the components $\mathbf{x}(1)=[m G], \mathbf{x}(2)=\left[m G^{*}\right], \mathbf{x}(3)=[t G], \mathbf{x}(4)=\left[t G^{*}\right], \mathbf{x}(5)=$ $[t G E F], \mathbf{x}(6)=\left[t G E F^{*}\right], \mathbf{x}(7)=[m G A P]$, and $\mathbf{x}(8)=\left[m G A P^{*}\right]$. We also write the reaction velocities as

$$
\begin{aligned}
& v_{1}=\mathbf{x}(1), v_{2}=\rho_{o f f}^{m G} \mathbf{x}(2) \mathbf{x}(8), v_{3}=\rho_{o n}^{I} \mathbf{x}(2) \mathbf{x}(5), v_{4}=\rho_{o n}^{t G} \mathbf{x}(3) \mathbf{x}(6) \\
& v_{5}=\rho_{o f f}^{m G} \mathbf{x}(4), v_{6}=\rho_{o n}^{I I} \mathbf{x}(6) \mathbf{x}(7), v_{7}=\rho_{o n}^{I I I} \mathbf{x}(4) \mathbf{x}(7) .
\end{aligned}
$$

The $8 \times 7$ stoichiometric matrix for the system given by Eqs. $2.1-2.8$ is then given by

$$
S=\left[\begin{array}{cccccccc} 
& \multicolumn{9}{c}{\text { arrow 1 }} & \multicolumn{1}{c}{\text { arrow 2 }} & \text { arrow 3 } \\
\mathrm{mG} & -1 & 1 & 0 & 0 & 0 & 0 & 0 \\
\mathrm{mG}^{*} & 1 & -1 & -1 & 0 & 0 & 0 & 0 \\
\mathrm{tG} & 0 & 0 & 0 & -1 & 1 & 0 & 0 \\
\mathrm{tG} & 0 & 0 & 0 & 1 & -1 & 0 & -1 \\
\mathrm{tGEF} & 0 & 0 & -1 & 0 & 0 & 0 & 0 \\
\mathrm{tGEF} * & 0 & 0 & 1 & 0 & 0 & -1 & 0 \\
\mathrm{mGAP}^{*} & 0 & 0 & 0 & 0 & 0 & -1 & -1 \\
\mathrm{mGAP}^{*} & 0 & 0 & 0 & 0 & 0 & 1 & 1
\end{array}\right]
$$

where the rows and columns of $S$ (Eq. 3.1) represent the 8 components and 7 reactions, respectively. The right null space of $S$ comprises the steady state flux solutions, and the left null space contains the conservation laws of the system [69]. On the other hand, the column space contains the dynamics of the time-derivatives, and the rank of $\mathrm{S}$ is the actual dimension of the system in which the dynamics take place. In the following subsections, we assume that $\rho_{o f f}^{m G}, \rho_{o n}^{t G}$, and $\rho_{\text {off }}^{t G}$ are strictly positive and we analyze Eqs. 2.9 - 2.16 when the mGTPase and tGTPase switches are coupled through: (i) A forward coupling $m G^{*} \rightarrow t G E F$ only (arrow 1), (ii) forward coupling $m G^{*} \rightarrow t G E F$ and feedback loop $t G E F \rightarrow m G A P$ (arrows 1 and 2) and (iii) forward coupling connection $m G^{*} \rightarrow t G E F$ and feedback loops $t G E F \rightarrow m G A P$ and $t G^{*} \rightarrow m G A P$ (arrows 1,2 , and 3). While mathematically, other combinations of arrows are possible, biologically, these are the only relevant combinations either for cellular function or for experimental manipulation.

\subsection{Forward coupling connection: Recruitment of tGEF by active mGTPases $\left(m G^{*} \rightarrow\right.$}

$t G E F$ ). To analyze Eqs. $2.9-2.16$ with the forward coupling connection only (arrow 1 in Fig.1C), we assume $\rho_{\text {on }}^{I}>0$ and $\rho_{\text {on }}^{I I}=\rho_{\text {on }}^{I I I}=0$, which means that the feedback 
loops (arrows 2 and 3) are not considered this first analysis. This represents the simple connection of the two GTPase switches, which, in cells appears to be mediated via activation-dependent coupling of $\mathrm{mG}^{*}$ to tGEF [32]. In this case, the stoichiometric matrix (Eq. 3.1) is $8 \times 5$.

Conservation laws. For this particular system, the total concentrations $\left[t G_{t o t}\right]:=[t G]+$ $\left[t G^{*}\right]$ and $\left[t G E F_{t o t}\right]:=[t G E F]+\left[t G E F^{*}\right]$ are constant over time and are strictly positive. For this reason, it is convenient to introduce the fractions $\mathcal{T}:=\frac{[t G]}{\left[t G_{t o t}\right]}$ and $\mathcal{G}:=\frac{[t G E F]}{\left[t G E F_{t o t}\right]}$ of inactive tGTPase and tGEF in the system, respectively, and let $\mathcal{T}^{*}$ and $\mathcal{G}^{*}$ denote the fraction of their active forms. We then use $\mathcal{T}+\mathcal{T}^{*}=1$ and $\mathcal{G}+\mathcal{G}^{*}=1$ to rewrite the system in the form

$$
\begin{aligned}
\frac{d[m G]}{d t} & =-[m G]+\rho_{o f f}^{m G}\left[m G A P^{*}\right]\left[m G^{*}\right] \\
\frac{d\left[m G^{*}\right]}{d t} & =[m G]-\rho_{o f f}^{m G}\left[m G A P^{*}\right]\left[m G^{*}\right]-\rho_{\text {on }}^{I}\left[t G E F_{\text {tot }}\right]\left(1-\mathcal{G}^{*}\right)\left[m G^{*}\right] \\
\frac{d \mathcal{T}^{*}}{d t} & =\rho_{\text {on }}^{t G}\left[t G E F_{\text {tot }}\right] \mathcal{G}^{*}\left(1-\mathcal{T}^{*}\right)-\rho_{\text {off }}^{t G} \mathcal{T}^{*} \\
\frac{d \mathcal{G}^{*}}{d t} & =\rho_{\text {on }}^{I}\left(1-\mathcal{G}^{*}\right)\left[m G^{*}\right]
\end{aligned}
$$

From the stoichiometric matrix (Eq. 3.1), we observe that $[m G]+\left[m G^{*}\right]+\left[t G E F^{*}\right]=C$, where $C>0$ is constant over time, and thus

$$
[m G]+\left[m G^{*}\right]+\left[t G E F_{t o t}\right] \mathcal{G}^{*}=C
$$

follows by the definition of $\mathcal{G}^{*}$. We compute the left null space of the stoichiometric matrix and confirm the total of three conservation laws in this case. The conservation law given by Eq. 3.6 reduces the system to three unknowns, which eases the steady state and stability analysis.

Steady states. To find biologically plausible (nonnegative) steady states of the system given by Eqs. $3.2-3.6$, we must find $\widehat{[m G]}, \widehat{\left[m G^{*}\right]}, \widehat{\mathcal{G}^{*}}$, and $\widehat{\mathcal{T}^{*}}$ such that the timederivatives in Eqs. 3.2-3.5 are zero and the conservation law given by Eq. 3.6 is satisfied. Therefore, we must solve the following system:

$$
\begin{aligned}
& \widehat{[m G]}-\rho_{o f f}^{m G}\left[m G A P^{*}\right] \widehat{\left[m G^{*}\right]}-\rho_{\text {on }}^{I}\left[t G E F_{t o t}\right]\left(1-\widehat{\mathcal{G}^{*}}\right) \widehat{\left[m G^{*}\right]}=0 \\
& \rho_{\text {on }}^{t G}\left[t G E F_{t o t}\right] \widehat{\mathcal{G}^{*}}\left(1-\widehat{\mathcal{T}^{*}}\right)-\rho_{o f f}^{t G} \widehat{\mathcal{\mathcal { T }}^{*}}=0 \\
& \left(1-\widehat{\mathcal{G}^{*}}\right) \widehat{\left[m G^{*}\right]}=0 \\
& \widehat{[m G]}+\widehat{\left[m G^{*}\right]}+\left[t G E F_{t o t}\right] \widehat{\mathcal{G}^{*}}=C
\end{aligned}
$$

From the third equation above, we must have $\widehat{\left[m G^{*}\right]}=0$ or $\widehat{\mathcal{G}^{*}}=1$. Thus we divide the steady state analysis in these two cases and summarize our results in the following proposition, whose proof can be found in the appendix A. 
Proposition 3.1. The steady states $\widehat{\mathbf{x}}=\left(\widehat{[m G]}, \widehat{\left[m G^{*}\right]}, \widehat{\mathcal{T}^{*}}, \widehat{\mathcal{G}^{*}}\right)$ of the system given by Eqs. $3.2-3.6$ are given by

- Steady state 1:

$$
\widehat{\mathbf{x}}=\left(0,0, \frac{1}{1+\frac{\rho_{o f f}^{t G}}{\rho_{o n}^{t G C}}}, \frac{C}{\left[t G E F_{t o t}\right]}\right)
$$

if and only if $C \leq\left[t G E F_{\text {tot }}\right]$ and

- Steady state 2:

$$
\begin{aligned}
\widehat{\mathbf{x}}= & \left(\frac{\rho_{o f f}^{m G}\left[m G A P^{*}\right]}{1+\rho_{o f f}^{m G}\left[m G A P^{*}\right]}\left(C-\left[t G E F_{t o t}\right]\right),\right. \\
& \frac{C-\left[t G E F_{t o t}\right]}{1+\rho_{o f f}^{m G}\left[m G A P^{*}\right]}, \\
& \left.\frac{\rho_{o n}^{t G}\left[t G E F_{t o t}\right]}{\rho_{o n}^{t G}\left[t G E F_{t o t}\right]+\rho_{o f f}^{t G}}, 1\right) .
\end{aligned}
$$

if and only if $C \geq\left[t G E F_{\text {tot }}\right]$.

Given the explicit expressions for the steady states and the parameter range in which they exist, we perform a local stability analysis to determine if these states are stable or unstable under small perturbations. We adopt the classical linearization procedure based on the powerful Hartman-Grobman theorem [61, 62]. We show that the steady states are locally asymptotically stable, which means that any trajectory will be attracted to the steady state provided the initial condition is sufficiently close.

Local Stability Analysis. Using that $[m G]=C-\left[m G^{*}\right]-\left[t G E F_{t o t}\right] \mathcal{G}^{*}$ (from Eq. 3.6) in Eqs. 3.2 - 3.5, we obtain the following three-dimensional system:

$$
\begin{aligned}
\frac{d\left[m G^{*}\right]}{d t} & =f_{1}\left(\left[m G^{*}\right], \mathcal{G}^{*}, \mathcal{T}^{*}\right) \\
\frac{d \mathcal{T}^{*}}{d t} & =f_{2}\left(\left[m G^{*}\right], \mathcal{G}^{*}, \mathcal{T}^{*}\right) \\
\frac{d \mathcal{G}^{*}}{d t} & =f_{3}\left(\left[m G^{*}\right], \mathcal{G}^{*}, \mathcal{T}^{*}\right)
\end{aligned}
$$

where

$$
\begin{aligned}
f_{1}\left(\left[m G^{*}\right], \mathcal{G}^{*}, \mathcal{T}^{*}\right) & =\left(C-\left[m G^{*}\right]-\left[t G E F_{\text {tot }}\right] \mathcal{G}^{*}\right) \\
& -\rho_{\text {off }}^{m G}\left[m G A P^{*}\right]\left[m G^{*}\right]-\rho_{\text {on }}^{I}\left[t G E F_{\text {tot }}\right]\left(1-\mathcal{G}^{*}\right)\left[m G^{*}\right] \\
f_{2}\left(\left[m G^{*}\right], \mathcal{G}^{*}, \mathcal{T}^{*}\right) & =\rho_{\text {on }}^{t G}\left[t G E F_{\text {tot }}\right] \mathcal{G}^{*}\left(1-\mathcal{T}^{*}\right)-\rho_{\text {off }}^{t G}\left[t G A P^{*}\right] \mathcal{T}^{*}
\end{aligned}
$$


and

$$
f_{3}\left(\left[m G^{*}\right], \mathcal{G}^{*}, \mathcal{T}^{*}\right)=\rho_{o n}^{I}\left(1-\mathcal{G}^{*}\right)\left[m G^{*}\right]
$$

To perform the local stability analysis, we calculate the Jacobian matrix evaluated at the steady state

$$
\mathcal{J}\left[\widehat{\left[m G^{*}\right]}, \widehat{\mathcal{T}^{*}}, \widehat{\mathcal{G}^{*}}\right]=\left.\left[\begin{array}{lll}
\frac{\partial f_{1}}{\partial\left[m G^{*}\right]} & \frac{\partial f_{1}}{\partial \mathcal{T}^{*}} & \frac{\partial f_{1}}{\partial \mathcal{G}^{*}} \\
\frac{\partial f_{2}}{\partial\left[m G^{*}\right]} & \frac{\partial f_{2}}{\partial \mathcal{T}^{*}} & \frac{\partial f_{2}}{\partial \mathcal{G}^{*}} \\
\frac{\partial f_{3}}{\partial\left[m G^{*}\right]} & \frac{\partial f_{3}}{\partial \mathcal{T}^{*}} & \frac{\partial f_{3}}{\partial \mathcal{G}^{*}}
\end{array}\right]\right|_{\left(\widehat{\left.m G^{*}\right]}, \widehat{\mathcal{T}^{*}}, \widehat{\mathcal{G}^{*}}\right)}
$$

and by showing that all its eigenvalues have a negative real part, we can prove that the steady state is LAS [61], provided we further assume that the strict inequalities from Proposition 3.1 hold. This is the content of the following theorem.

Theorem 3.1. Let $C$ be the conservation quantity from $E q$. 3.6. Then,

(1) If $C<\left[t G E F_{\text {tot }}\right]$, the steady state 1 (Eq. 3.7) is LAS.

(2) If $C>\left[t G E F_{\text {tot }}\right]$, the steady state 2 (Eq. 3.8) is LAS.

Proof. All calculations were done with MATLAB's R2019b symbolic toolbox using the functions jacobian and eig to compute the Jacobian matrices and their eigenvalues, respectively. We proceed with the analysis of each case separately.

(1) Suppose $C<\left[t G E F_{t o t}\right]$. As we have seen in the previous subsection, in this case the steady state is given by Eq. 3.7. The Jacobian matrix (Eq. 3.9) is given by

$$
\left[\begin{array}{ccc}
\rho_{o n}^{I}\left(C-\left[t G E F_{t o t}\right]\right)-1-\left[m G A P^{*}\right] \rho_{o f f}^{m G} & 0 & -\left[t G E F_{t o t}\right] \\
0 & -C \rho_{o n}^{t G}-\rho_{o f f}^{t G} & \frac{\rho_{o f f}^{t G} f_{o n}^{t G}\left[t G E F_{t o t}\right]}{C \rho_{o n}^{t+G} \rho_{o f f}^{t G}} \\
-\rho_{o n}^{I}\left(\frac{C}{\left[t G E F_{t o t}\right]}-1\right) & 0 & 0
\end{array}\right] .
$$

The first eigenvalue in this case is given by $\lambda_{1}=-C \rho_{o n}^{t G}-\rho_{o f f}^{t G}$ and the other two $\left(\lambda_{2}\right.$ and $\left.\lambda_{3}\right)$ are such that

$$
\lambda_{2}+\lambda_{3}=\rho_{o n}^{I}\left(C-\left[t G E F_{t o t}\right]\right)-\rho_{o f f}^{m G}\left[m G A P^{*}\right]-1<0
$$

and

$$
\lambda_{2} \lambda_{3}=-\rho_{o n}^{I}\left(C-\left[t G E F_{t o t}\right]\right)>0
$$

from which we conclude that $\lambda_{2}$ and $\lambda_{3}$ are both negative and therefore the steady state is LAS. 
(2) Suppose now that $C>\left[t G E F_{t o t}\right]$. Following our previous analysis, the steady state is given by Eq. 3.8. The Jacobian matrix in this case is given by

$$
\left[\begin{array}{ccc}
-\rho_{o f f}^{m G}\left[m G A P^{*}\right]-1 & 0 & {\left[t G E F_{t o t}\right]\left(\frac{\rho_{o n}^{I}\left(C-t G E F_{t o t}\right)}{\rho_{o f f}^{m G}\left[m G A P^{*}\right]+1}-1\right)} \\
0 & -\rho_{o f f}^{t G}-\rho_{o n}^{t G}\left[t G E F_{t o t}\right] & \frac{\rho_{o f f}^{t G} \rho_{o n}^{t G}\left[t G E F_{t o t}\right]}{\rho_{o f f}^{t G}+\rho_{o n}^{t G}\left[t G E F_{t o t}\right]} \\
0 & 0 & -\frac{\rho_{o n}^{I}\left(C-\left[t G E F_{t o t}\right]\right)}{\rho_{o f f}^{m G}\left[m G A P^{*}\right]+1}
\end{array}\right]
$$

and the eigenvalues are given by $\lambda_{1}=-\rho_{o f f}^{t G}-k_{o n}^{t G}\left[t G E F_{t o t}\right], \lambda_{2}=-1-$ $\rho_{o f f}^{m G}\left[m G A P^{*}\right]$ and $\lambda_{3}=-\frac{\rho_{o n}^{I}\left(C-\left[t G E F_{t o t}\right]\right)}{\rho_{o f f}^{m G}\left[m G A P^{*}\right]+1}$, which are all negative and this completes the proof.

The inequality $C<\left[t G E F_{\text {tot }}\right]$ must hold for existence and local asymptotic stability to steady state 1 . Recalling the definition of $\mathcal{G}^{*}$ and that Eq. 3.6 holds for all times, including $t=0$, this relationship between $C$ and $\left[t G E F_{t o t}\right]$ can be rewritten as $[m G](0)+\left[m G^{*}\right](0)<[t G E F](0)$ where $[t G E F](0)=\left[t G E F_{t o t}\right]-\left[t G E F^{*}\right](0)$ is initial concentration of cytosolic tGEF that is yet to be recruited by $\mathrm{mG}^{*}$ to the membranes. Similarly, the steady state 2 will exist when $[m G](0)+\left[m G^{*}\right](0)>[t G E F](0)$. In this case, the reduced system will converge to a state where some distribution of $\mathrm{mG}$, $\mathrm{mG}^{*}, \mathrm{tG}, \mathrm{tG}^{*}$ are present (Eq. 3.8), given sufficiently close initial and steady state concentration values. Thus, the existence of the steady states depends only on the initial concentrations and not on any kinetic parameters.

Fig 1(E) illustrates the two possible steady states (gray-colored "1" in the $2 \times 2$ table) promoted by the coupling connection. steady state 1 can be interpreted as a configuration where the copy numbers of both active and inactive mGTPase are low, while the tGTPase copy numbers remain high. On the other hand, in steady state 2 both $\mathrm{m}$ - and tGTPases have high copy numbers in both their active and inactive forms. Our results suggest that the coupling from $\mathrm{mG}$ to $\mathrm{tGEF}$, which initiates the coupling between the two G protein switches, can drive the system to two possible configurations depending on the cellular concentrations of total $\mathrm{mG}$ and tGEF. If the initial tGEF is larger than the total $\mathrm{mG}$, the coupling connection will result in a significant decrease of the total $\mathrm{mG}$ and result in the activation of a fraction of the $\operatorname{tGEF}\left(\widehat{\mathcal{G}^{*}}=\frac{C}{\left[t G E F_{t o t}\right]}\right.$ in Eq. 3.7). On the contrary, if the initial tGEF is less than the total $\mathrm{mG}$, then the available tGEF will be fully engaged $\left(\widehat{\mathcal{G}^{*}}=1\right.$ in Eq. 3.8$)$ and there will be a residual $\mathrm{mG}$ concentration in the system. We conclude that the initial difference between the copy numbers of total $\mathrm{mG}$ and tGEF (a cytosolic protein that is recruited to the membrane by $\mathrm{mG}^{*}$ ) is the main factor that will determine the steady state of the coupled GTPase switches. 


\subsection{Coupled switches with feedback loop $t G E F \rightarrow m G A P$ : Recruitment of tGEF} by active mGTPases and tGEF colocalization with mGAP. We analyze the case where the feedback loop $t G E F \rightarrow m G A P$ (arrow 2 in Fig.1C) is added to the coupled system with the forward connection. In cells, this feedback loop represents a tGEF* colocalization with mGAP on Golgi membranes that facilitates the recruitment of GAP proteins Fig.1A, [32]. To analyze the effects of Arrows 1 and 2 solely, we thus assume $\rho_{\text {on }}^{I}>0, \rho_{\text {on }}^{I I}>0$ and $\rho_{\text {on }}^{I I I}=0$. The model equations are thus given by the following system:

$$
\begin{aligned}
\frac{d[m G]}{d t} & =-[m G]+\rho_{o f f}^{m G}\left[m G A P^{*}\right]\left[m G^{*}\right] \\
\frac{d\left[m G^{*}\right]}{d t} & =[m G]-\rho_{o f f}^{m G}\left[m G A P^{*}\right]\left[m G^{*}\right]-\rho_{o n}^{I}[t G E F]\left[m G^{*}\right] \\
\frac{d[t G]}{d t} & =-\rho_{o n}^{t G}\left[t G E F^{*}\right][t G]+\rho_{o f f}^{t G}\left[t G^{*}\right] \\
\frac{d\left[t G^{*}\right]}{d t} & =\rho_{o n}^{t G}\left[t G E F^{*}\right][t G]-\rho_{o f f}^{t G}\left[t G^{*}\right] \\
\frac{d[t G E F]}{d t} & =-\rho_{o n}^{I}[t G E F]\left[m G^{*}\right] \\
\frac{d\left[t G E F^{*}\right]}{d t} & =\rho_{o n}^{I}[t G E F]\left[m G^{*}\right]-\rho_{\text {on }}^{I I}\left[t G E F^{*}\right][m G A P] \\
\frac{d[m G A P]}{d t} & =-\rho_{\text {on }}^{I I}[m G A P]\left[t G E F^{*}\right] \\
\frac{d\left[m G A P^{*}\right]}{d t} & =\rho_{o n}^{I I}[m G A P]\left[t G E F^{*}\right]
\end{aligned}
$$

As in Section 3.1, we first analyze the conservation laws of this particular system. In this case, the stoichiometric matrix (Eq. 3.1) is $8 \times 6$.

Conservation Laws. We begin by observing that the total amount of tGTPase is conserved in this system. Thus we may use the fraction $\mathcal{T}^{*}$ as in Section 3.1 and that is the first conservarion law. The total amount of mGAP is also conserved, as we sum Eqs. 3.16 and 3.17. We can then write

$$
[m G A P]=\left[m G A P_{t o t}\right]-\left[m G A P^{*}\right]
$$

and substitute the above expression for $[m G A P]$ in Eqs. 3.15 and 3.17. We choose to keep the concentrations of $\mathrm{mGAP}$ as a variable for notational simplicity and do not define its fraction. Summing Eqs. 3.10, 3.11, 3.15, and 3.17, and integrating over time, we get

$$
[m G]+\left[m G^{*}\right]+\left[t G E F^{*}\right]+\left[m G A P^{*}\right]=C_{1}
$$

where $C_{1} \geq 0$ is constant over time. Moreover, Eqs. 3.14, 3.15, and 3.17 when summed and integrated give

$$
[t G E F]+\left[t G E F^{*}\right]+\left[m G A P^{*}\right]=C_{2}
$$


for $C_{2} \geq 0$ also constant. We compute the left null space of the stoichiometric matrix (Eq. 3.1) and confirm a total of four conservation laws in this case. The reduced system is given by the following equations:

$$
\begin{aligned}
\frac{d[m G]}{d t} & =-[m G]+\rho_{o f f}^{m G}\left[m G A P^{*}\right]\left[m G^{*}\right] \\
\frac{d\left[m G^{*}\right]}{d t} & =[m G]-\rho_{o f f}^{m G}\left[m G A P^{*}\right]\left[m G^{*}\right]-\rho_{o n}^{I}[t G E F]\left[m G^{*}\right] \\
\frac{d \mathcal{T}^{*}}{d t} & =\rho_{o n}^{t G}\left[t G E F^{*}\right]\left(1-\mathcal{T}^{*}\right)-\rho_{o f f}^{t G} \mathcal{T}^{*} \\
\frac{d[t G E F]}{d t} & =-\rho_{o n}^{I}[t G E F]\left[m G^{*}\right] \\
\frac{d\left[t G E F^{*}\right]}{d t} & =\rho_{o n}^{I}[t G E F]\left[m G^{*}\right]-\rho_{o n}^{I I}\left[t G E F^{*}\right]\left(\left[m G A P_{t o t}\right]-\left[m G A P^{*}\right]\right) \\
\frac{d\left[m G A P^{*}\right]}{d t} & =\rho_{o n}^{I I}\left[t G E F^{*}\right]\left(\left[m G A P_{t o t}\right]-\left[m G A P^{*}\right]\right)
\end{aligned}
$$

with the conservation laws given by Eqs. 3.19 and 3.20. Next, we obtain the steady states of the system.

Steady states and local stability analysis. To find the steady states, we must find nonnegative solutions of the following system:

$$
\begin{aligned}
& -\widehat{[m G]}+\rho_{o f f}^{m G}\left[\widehat{m G A P^{*}}\right] \widehat{\left[m G^{*}\right]}=0 \\
& \rho_{\text {on }}^{t G}\left[{\widehat{t G E F^{*}}}^{*}\left(1-\widehat{\mathcal{T}}^{*}\right)-\rho_{\text {off }}^{t G} \widehat{\mathcal{T}}^{*}=0\right. \\
& {[\widehat{t G E F}] \widehat{\left[m G^{*}\right]}=0} \\
& \left(\left[m G A P_{t o t}\right]-\left[\widehat{m G A P}^{*}\right]\right)\left[\widehat{t G E F}^{*}\right]=0 \\
& \widehat{[m G]}+\widehat{\left[m G^{*}\right]}+\left[t \widehat{t G E F^{*}}\right]+\left[\widehat{m G A P}^{*}\right]=C_{1} \\
& {[\widehat{t G E F}]+\left[\widehat{t G E F}^{*}\right]+\left[\widehat{m G A P}^{*}\right]=C_{2}}
\end{aligned}
$$

From Eq. 3.29, we must have $[\widehat{t G E F}]=0$ or $\widehat{\left[m G^{*}\right]}=0$. Moreover, from Eq. 3.30, $\left[\widehat{t G E F}^{*}\right]=0$ or $\left[\widehat{m G A P}^{*}\right]=\left[m G A P_{t o t}\right]$ and thus we have four possible combinations to analyze.

We study each case separately and obtain the necessary and sufficient inequalities involving the parameters $C_{1}, C_{2}$, and $\left[m G A P_{t o t}\right]$ that ensure the existence of each steady state. As in Section 3.1, we also show that the steady states are LAS provided the strict inequalities are satisfied. We summarize our analysis in the following theorem, whose proof can be found in Appendix B. 
Theorem 3.2. The steady states

$$
\widehat{\mathbf{x}}=\left(\widehat{[m G]}, \widehat{\left[m G^{*}\right.}\right], \widehat{\mathcal{T}^{*}},[\widehat{t G E F}],\left[\widehat{t G E F^{*}}\right],\left[{\widehat{m G A P^{*}}}^{*}\right)
$$

of the system given by Eqs. 3.19 - 3.26 are given by

- Steady state 1:

$$
\widehat{\mathbf{x}}=\left(0,0, \frac{\rho_{o n}^{t G}\left(C_{1}-\left[m G A P_{t o t}\right]\right)}{\rho_{o n}^{t G}\left(C_{1}-\left[m G A P_{t o t}\right]\right)+\rho_{o f f}^{t G}}, C_{2}-C_{1}, C_{1}-\left[m G A P_{t o t}\right],\left[m G A P_{t o t}\right]\right)
$$

if and only if $C_{2} \geq C_{1}$ and $C_{1} \geq\left[m G A P_{t o t}\right]$. The steady state is LAS if $C_{2}>C_{1}$ and $C_{1}>\left[m G A P_{t o t}\right]$.

- Steady state 2:

$$
\begin{aligned}
\widehat{\mathbf{x}}= & \left(\frac{\rho_{o f f}^{m G}\left[m G A P_{t o t}\right]}{1+\rho_{o f f}^{m G}\left[m G A P_{t o t}\right]}\left(C_{1}-C_{2}\right), \frac{\left(C_{1}-C_{2}\right)}{1+\rho_{o f f}^{m G}\left[m G A P_{t o t}\right]},\right. \\
& \left.\frac{\rho_{o n}^{t G}\left(C_{2}-\left[m G A P_{t o t}\right]\right)}{\left(C_{2}-\left[m G A P_{t o t}\right]\right)+\rho_{o f f}^{t G}}, 0, C_{2}-\left[m G A P_{t o t}\right],\left[m G A P_{t o t}\right]\right)
\end{aligned}
$$

if and only if $C_{1} \geq C_{2}$ and $C_{2} \geq\left[m G A P_{t o t}\right]$. The steady state is LAS if $C_{1}>C_{2}$ and $C_{2}>\left[m G A P_{t o t}\right]$.

- Steady state 3:

$$
\widehat{\mathbf{x}}=\left(0,0,0, C_{2}-C_{1}, 0, C_{1}\right)
$$

if and only if $C_{2} \geq C_{1}$ and $C_{1} \leq\left[m G A P_{t o t}\right]$. The steady state is $L A S$ if $C_{2}>C_{1}$ and $C_{1}<\left[m G A P_{t o t}\right]$.

- Steady state 4:

$$
\widehat{\mathbf{x}}=\left(\frac{1}{1+\rho_{o f f}^{m G} C_{2}}\left(C_{1}-C_{2}\right), 0,0,0, C_{2}\right)
$$

if and only if $C_{1} \geq C_{2}$ and $C_{2} \leq\left[m G A P_{t o t}\right]$. The steady state is $L A S$ if $C_{1}>C_{2}$ and $C_{2}<\left[m G A P_{t o t}\right]$.

Recalling the definitions of $C_{1}$ and $C_{2}$ and the fact that Eqs. 3.19 and 3.20 hold at all times, including at $t=0$, we can write $C_{1}=[m G](0)+\left[m G^{*}\right](0)+\left[t G E F^{*}\right](0)+$ $\left[m G A P^{*}\right](0)$ and $C_{2}=[t G E F](0)+\left[t G E F^{*}\right](0)+\left[m G A P^{*}\right](0)$. In this way, from the inequalities obtained in Theorem 3.2 for $C_{1}$ and $C_{2}$, we obtain relationships among the initial conditions of the original system (Eqs. $3.10-3.17$ ) that are associated with each one of the four steady states.

For the existence and local asymptotic stability of steady state 1 (Eq. 3.33), where $\mathrm{mG}$ and $\mathrm{mG}^{*}$ have zero concentration values, the inequalities $C_{2}>C_{1}$ and $C_{1}>\left[m G A P_{t o t}\right]$ must hold. The first inequality can be written as $[m G](0)+\left[m G^{*}\right](0)<[t G E F](0)$, which was obtained in Section 3.1 as the existence condition for the steady state with no $\mathrm{mG}$ and $\mathrm{mG}^{*}$ (Eq. 3.7). On the other hand, the inequality $C_{1}>\left[m G A P_{\text {tot }}\right]$ can be written as $[m G](0)+\left[m G^{*}\right](0)+\left[t G E F^{*}\right](0)>[m G A P](0)$, where $[m G A P](0)$ is the initial concentration of cytosolic mGAP that is yet to be recruited by tGEF* to the 
membranes. Therefore, two conditions guarantee the existence of steady state 1: (1) The total amount of $\mathrm{mG}$ protein must be initially less than the concentration of tGEF and (2) The sum of the concentrations of total $\mathrm{mG}$ protein and $\mathrm{tGEF}^{*}$ must be initially higher than the concentration of mGAP. If both conditions hold, then Theorem 3.2 ensures that steady state 1 will emerge and the reduced system (Eqs. $3.21-3.26$ along with Eqs. 3.19 and 3.20) will converge to the steady state 1 , provided the initial and steady state concentration values are sufficiently close.

A similar analysis holds for steady states 2, 3 and 4. For simplicity, we present the required initial conditions for each steady state without repeating the conclusions that follows from Theorem 3.2. For steady state 2 (Eq. 3.34), where $\mathrm{mG}, \mathrm{mG}^{*}$, $\mathrm{tG}$, and $\mathrm{tG}^{*}$ are present, the inequalities $C_{1}>C_{2}$ and $C_{2}>\left[m G A P_{t o t}\right]$ become $[m G](0)+\left[m G^{*}\right](0)>$ $[t G E F](0)$ and $[t G E F](0)+\left[t G E F^{*}\right](0)>[m G A P](0)$, respectively. Hence, the total amount of $\mathrm{mG}$ protein must be initially higher than the concentration of tGEF and the total amount of tGEF must be initially higher than concentration of mGAP. For steady state 3 (Eq. 3.35), where $\mathrm{mG}$ and $\mathrm{mG}^{*}$ have zero concentration values and the tGTPase is fully inactivated, the inequalities $C_{2}>C_{1}$ and $C_{1}<\left[m G A P_{\text {tot }}\right]$ become $[m G](0)+\left[m G^{*}\right](0)<[t G E F](0)$ and $[m G](0)+\left[m G^{*}\right](0)+\left[t G E F^{*}\right](0)<$ $[m G A P](0)$, respectively. Hence, the total amount of $\mathrm{mG}$ protein must be initially less than the concentration of tGEF and the sum of the concentrations of total $\mathrm{mG}$ protein and $\mathrm{tGEF}^{*}$ must be initially less than the concentration of mGAP. For steady state 4 (Eq. 3.36), where $\mathrm{mG}$ and $\mathrm{mG}^{*}$ are present and $\mathrm{tG}^{*}$ concentration is zero, the inequalities $C_{1}>C_{2}$ and $C_{2}<\left[m G A P_{t o t}\right]$ become $[m G](0)+\left[m G^{*}\right](0)>[t G E F](0)$ and $[t G E F](0)+\left[t G E F^{*}\right](0)<[m G A P](0)$, respectively. Hence, the total amount of $\mathrm{mG}$ protein must be initially higher than the concentration of tGEF and the total amount of tGEF must be initially less than the concentration of mGAP. In this case, the existence of the steady states also depends only on the initial concentrations and not on any kinetic parameters.

Fig 1(E) illustrates the four possible steady states (gray-colored " $1+2$ " in the $2 \times 2$ table) promoted by the coupled switches in the presence of the feedback loop $t G E F \rightarrow$ $m G A P$. Steady states 1 and 2 have the same interpretation of the two steady states obtained in Section 3.1. On the other hand, steady states 3 and 4 were obtained through the sole contribution of the feedback loop $t G E F \rightarrow m G A P$. These states share the common feature of having tGTPase fully inactivated. However, steady state 3 can be interpreted as a configuration where the copy numbers of $\mathrm{mG}$ and $\mathrm{mG}^{*}$ are low, while in steady state 4 , these copy numbers are high.

3.3. Coupled switches with feedback loops $t G E F \rightarrow m G A P$ and $t G^{*} \rightarrow m G A P$ : Recruitment of tGEF by active mGTPases, tGEF colocalization with mGAP, and activation of mGAP by active tGTPases. We analyze the case where the feedback loop $t G^{*} \rightarrow m G A P$ (arrow 3 in Fig.1C) is added to the coupled system in addition 
to the feedback loop $t G E F \rightarrow m G A P$. This connection represents the release of free $G \beta \gamma$ promoting mGAP activation. We analyze the full system given by Eqs. 2.9 2.16 in the case where $\rho_{\text {on }}^{I}, \rho_{o n}^{I I}$, and $\rho_{\text {on }}^{I I I}$ are strictly positive. In particular, we obtain the conservation laws and four 1-parameter steady state families. We also obtain the necessary conditions for the conserved quantities that guarantee the existence of each steady state family.

Conservation Laws. As in Section 3.2, we observe that the total amount of mGAP is constant over time, so Eq. 3.18 still holds. On the other hand, the total tGTPase follows a new conservation law that we derive here. Summing Eqs. $2.9-2.12,2.14$, and 2.16 and integrating over time, we have

$$
[m G]+\left[m G^{*}\right]+[t G]+\left[t G^{*}\right]+\left[t G E F^{*}\right]+\left[m G A P^{*}\right]=\tilde{C}_{1} .
$$

Summing Eqs. 2.11 - 2.14 and Eq. 2.16 and integrating over time, we obtain

$$
[t G]+\left[t G^{*}\right]+[t G E F]+\left[t G E F^{*}\right]+\left[m G A P^{*}\right]=\tilde{C}_{2}
$$

where $\tilde{C}_{1}$ and $\tilde{C}_{2}$ must be nonnegative constants. We compute the left null space of the stoichiometric matrix (Eq. 3.1) and confirm the total of three conservation laws, which are given by Eqs. 3.18, 3.37, and 3.38. These equations reduce Eqs. $2.9-2.16$ to a five-dimensional system, whose steady states can be obtained.

Steady states. We compute the steady states of the system when the time derivatives in Eqs. $2.9-2.16$ are equal to zero. Removing the linearly dependent equations, the problem reduces to finding the nonnegative solutions of the following system:

$$
\begin{aligned}
-\widehat{m G}]+\rho_{o f f}^{m G}\left[\widehat{\left.m G A P^{*}\right]}\right] \widehat{\left[m G^{*}\right]} & =0 \\
-\rho_{o n}^{t G}\left[\widehat{t G E F^{*}}\right] \widehat{[t G]}+\rho_{o f f}^{t G} \widehat{\left[t G^{*}\right]} & =0 \\
\left(\left[m G A P_{t o t}\right]-\left[\widehat{\left.m G A P^{*}\right]}\right] \widehat{\left[t G^{*}\right]}\right. & =0 \\
{[\widehat{t G E F}] \widehat{\left[m G^{*}\right]} } & =0 \\
{\left[{ \widehat { t G E F ^ { * } } ] } ^ { * } \left(\left[m G A P_{t o t}\right]-\left[\widehat{\left.m G A P^{*}\right]}\right)\right.\right.} & =0
\end{aligned}
$$

along with the conservation laws given by Eqs. 3.18, 3.37, and 3.38.

Eq. 3.40 gives $\widehat{\left[t G^{*}\right]}=\frac{\rho_{o n}^{t G}\left[t \widehat{\left.G E F^{*}\right][t G]}\right.}{\rho_{o f f}^{t G}}$ and Eq. 3.41 then becomes

$$
\left(\left[m G A P_{t o t}\right]-\left[\widehat{m G A P}^{*}\right]\right)\left[\widehat{t G E F^{*}}\right] \widehat{[t G]}=0
$$

From Eq. 3.43, we conclude that $\widehat{[t G]}$ can be any nonnegative real number satisfying Eqs. 3.37 and 3.38. We define $\xi=\widehat{[t G]}$ and characterize four $\xi$-dependent families of steady 
states similarly as we did in Section 3.2. We summarize our results in the following theorem, whose proof can be found in the appendix $\mathrm{C}$.

Theorem 3.3. The $\xi$-dependent families of steady states

$$
\widehat{\mathbf{x}}_{\xi}=\left(\widehat{[m G]}, \widehat{\left[m G^{*}\right]}, \widehat{[t G]}, \widehat{\left[t G^{*}\right]},[\widehat{t G E F}],\left[\widehat{t G E F^{*}}\right],\left[\widehat{m G A P^{*}}\right]\right)
$$

of the system given by Eqs. 3.39 - 3.43 with the conservation laws given by Eqs. 3.18, 3.37 , and 3.38 are given by

- Family 1:

$$
\begin{aligned}
\widehat{\boldsymbol{x}}_{\xi}= & \left(0,0, \xi, \frac{\left(\tilde{C}_{1}-\left[m G A P_{t o t}\right]-\xi\right) \rho_{o n}^{t G} \xi}{\rho_{o f f}^{t G}+\rho_{o n}^{t G} \xi}, \tilde{C}_{2}-\tilde{C}_{1},\right. \\
& \left.\left(\tilde{C}_{1}-\left[m G A P_{t o t}\right]-\xi\right) \frac{\rho_{o f f}^{t G}}{\rho_{o f f}^{t G}+\rho_{o n}^{t G \xi}},\left[m G A P_{t o t}\right]\right)
\end{aligned}
$$

only if $0 \leq \xi+\left[m G A P_{t o t}\right] \leq \tilde{C}_{1} \leq \tilde{C}_{2}$.

- Family 2:

$$
\begin{aligned}
\widehat{\boldsymbol{x}}_{\xi}= & \left(\frac{\left(\tilde{\tilde{C}_{1}}-\tilde{C}_{2}\right) \rho_{\text {off }}^{m G}\left[m G A P_{t o t}\right]}{1+\rho_{o f f}^{m G}\left[m G A P_{t o t}\right]}, \frac{\left(\tilde{C}_{1}-\tilde{C}_{2}\right)}{1+\rho_{o f f}^{m G}\left[m G A P_{t o t}\right]},\right. \\
& \left.\xi, \frac{\left.\left(\tilde{C}_{2}-\left[m G A P_{t o t}\right]-\xi\right]\right) \rho_{o n}^{t G} \xi}{\rho_{o f f}^{t G}+\rho_{o n}^{t G} \xi}, 0, \frac{\left.\left(\tilde{C}_{2}-\left[m G A P_{t o t}\right]-\xi\right]\right) \rho_{o f f}^{t G}}{\rho_{o f f}^{t G}+\rho_{o n}^{t G} \xi},\left[m G A P_{t o t}\right]\right)
\end{aligned}
$$

only if $0 \leq \xi+\left[m G A P_{t o t}\right] \leq \tilde{C}_{2} \leq \tilde{C}_{1}$.

- Family 3:

$$
\widehat{\mathbf{x}}_{\xi}=\left(0,0, \xi, 0, \tilde{C}_{2}-\tilde{C}_{1}, 0, \tilde{C}_{1}-\xi\right)
$$

only if $\max \left(0, \tilde{C}_{1}-\left[m G A P_{t o t}\right]\right) \leq \xi \leq \tilde{C}_{1} \leq \tilde{C}_{2}$.

- Family 4:

$$
\widehat{\boldsymbol{x}}_{\xi}=\left(\frac{\rho_{o f f}^{m G}\left(\tilde{C}_{1}-\tilde{C}_{2}\right)\left(\tilde{C}_{2}-\xi\right)}{1+\rho_{o f f}^{m G}\left(\tilde{C}_{2}-\xi\right)}, \frac{\left(\tilde{C}_{1}-\tilde{C}_{2}\right)}{1+\rho_{o f f}^{m G}\left(\tilde{C}_{2}-\xi\right)}, \xi, 0,0,0, \tilde{C}_{2}-\xi\right) .
$$

only if $\max \left(0, \tilde{C}_{2}-\left[m G A P_{t o t}\right]\right) \leq \xi \leq \tilde{C}_{2} \leq \tilde{C}_{1}$.

Recalling the definitions of $\tilde{C}_{1}$ and $\tilde{C}_{2}$ and the fact that Eqs. 3.37 and 3.38 hold at all times, including $t=0$, we can infer necessary relationships among the initial conditions for each steady state family. The inequality $\tilde{C}_{1} \leq \tilde{C}_{2}$ can be rewritten as $[m G](0)+$ $\left[m G^{*}\right](0) \leq[t G E F](0)$ is necessary for the emergence of Family 1 (Eq. 3.44) whith zero $\mathrm{mG}$ and $\mathrm{mG}^{*}$ values, which can be interpreted as a scenario in which nearly all the available $\mathrm{mG}$ proteins are activated to $\mathrm{mG}^{*}$, and that nearly all the $\mathrm{mG}^{*}$ species have successfully engaged with the available tGEFs, thereby maximally recruiting tGEF on the membranes. For Family 1, $\left[m G A P_{t o t}\right] \leq \tilde{C}_{1}$ also holds and can be written as $[m G A P](0) \leq$ $[m G](0)+\left[m G^{*}\right](0)+[t G](0)+\left[t G^{*}\right](0)+\left[t G E F^{*}\right](0)$, where $[m G A P](0)$ is the initial concentrations of cytosolic mGAP that is yet to be recruited by $\mathrm{tGEF}^{*}$ and $\mathrm{tG}^{*}$ to the 
membranes. Therefore, two initial conditions are necessary for the existence of Family 1: The total amount of $\mathrm{mG}$ protein must be initially less than the concentration of tGEF and (2) The summed concentrations of total $\mathrm{mG}$, total $\mathrm{tG}$ and $\mathrm{tGEF}^{*}$ must be initially higher than the concentration of mGAP. Finally, the inequality $0 \leq \xi+\left[m G A P_{t o t}\right] \leq \tilde{C}_{1}$ can be written as $0 \leq \xi \leq[m G](0)+\left[m G^{*}\right](0)+[t G](0)+\left[t G^{*}\right](0)+\left[t G E F^{*}\right](0)-[m G A P](0)$. Remarkably, we conclude that the initial balance between the summed concentrations of total $\mathrm{mG}$, total $\mathrm{tG}, \mathrm{tGEF}^{*}$ and the available mGAP is the upper bound for the $\mathrm{tG}$ concentration, which completely characterizes the necessary conditions for the emergence of Family 1.

A similar analysis can be done for Families 2, 3, and 4. For the existence of Family 2 (Eq. 3.45), where $\mathrm{mG}, \mathrm{mG}^{*} \mathrm{tG}, \mathrm{tG}^{*}$ are present (when $\xi>0$ ), the inequalities $\tilde{C}_{2} \leq$ $\tilde{C}_{1}$ and $\left[m G A P_{t o t}\right] \leq \tilde{C}_{2}$ must hold and can be rewritten as $[m G](0)+\left[m G^{*}\right](0) \geq$ $[t G E F](0)$ and $[m G A P](0) \leq[t G](0)+\left[t G^{*}\right](0)+[t G E F](0)\left[t G E F^{*}\right](0)$. Hence, the total amount of $\mathrm{mG}$ protein must be initially higher than the concentration of tGEF and the summed concentrations of total $\mathrm{tG}$ and total tGEF proteins must be initially higher than the concentration of mGAP. Finally, the inequality $0 \leq \xi+\left[m G A P_{t o t}\right] \leq \tilde{C}_{2}$ indicates that initial balance between the summed concentrations of total tG, total tGEF and the available mGAP is the upper bound for the $\mathrm{tG}$ concentrations. For Family 3 (Eq. 3.46, where $\mathrm{mG}$ and $\mathrm{mG}^{*}$ have zero concentration values and the tGTPase is fully inactivated, the inequality $\tilde{C}_{1} \leq \tilde{C}_{2}$ becomes $[m G](0)+\left[m G^{*}\right](0) \leq[t G E F](0)$. As for Family 1, the total amount of $\mathrm{mG}$ protein must be initially less than the concentration of tGEF. Moreover, from $\tilde{C}_{1}-\left[m G A P_{t o t}\right] \leq \xi$, the initial balance between the summed concentrations of total $\mathrm{mG}$, total $\mathrm{tG}, \mathrm{tGEF}^{*}$ and the available mGAP is the lower bound for the $\mathrm{tG}$ concentration. For Family 4 (Eq. 3.47), where $\mathrm{mG}$ and $\mathrm{mG}^{*}$ are present and tG* concentration is zero, $\tilde{C}_{2} \leq \tilde{C}_{1}$ becomes $[m G](0)+\left[m G^{*}\right](0) \geq[t G E F](0)$. As for Family, 2 the total amount of $\mathrm{mG}$ protein must be initially higher than the concentration of tGEF. Moreover, from $\tilde{C}_{2}-\left[m G A P_{t o t}\right] \leq \xi$, the initial balance between the summed concentrations of total tG, total tGEF and the available mGAP is the lower bound for the $\mathrm{tG}$ concentrations. As noted in the previous subsections, the existence of the steady states depends only on the initial concentrations and not on any kinetic parameters.

Fig 1(E) illustrates the four Families (gray-colored " $1+2+3$ " in the $2 \times 2$ table) promoted by the coupling connection $m G^{*} \rightarrow t G E F$ and the feedback loops $t G E F \rightarrow m G A P$ and $t G^{*} \rightarrow m G A P$. Families 1 and 2 have a similar interpretation of the steady states 1 and 2 obtained in Section 3.1 and Section 3.2. On the other hand, Families 3 and 4 were obtained through contributions of the feedback loop $t G^{*} \rightarrow m G A P$. These states share the common feature of having tGTPase fully inactivated. As for steady states 3 and 4, Family 3 can be interpreted as a configuration where the copy numbers of $\mathrm{mG}$ and $\mathrm{mG}^{*}$ are low, while in Family 4, those copy numbers are high. 
3.4. Numerical Simulations. To complete our mathematical analysis, we numerically investigate the range of initial conditions in which the trajectories of the original system (Eqs. $2.1-2.8$ ) converge to the different steady states. In particular, we illustrate the so-called basins of attraction [71] of the steady states, considering the same combination of connections between the two GTPase switches from $\S 3.1-\S 3.3$.

In Table 2, we describe each parameter of the system with the corresponding values that we used in our simulations. All ODEs were numerically solved in MATLAB R2018a with the function ode15s. The MATLAB codes can be downloaded in the link: https: //github.com/Rangamani-Lab/BMB_Matlab_codes.git

TABLE 2. Table of parameters and initial conditions

\begin{tabular}{|c|c|c|c|}
\hline Parameter & Meaning & Value/range & Unit \\
\hline$k_{o n}^{m G}$ & activation rate of the mGTPase & 3 (Figs 2,3,4, 5 A,B,C); $\mathcal{N}(30,1)$ (Fig 5 D,E,F) & $(s . \mu M)^{-1}$ \\
\hline$k_{o f f}^{m G}$ & deactivation rate of the mGTPase & 1 (Figs $2,3,4,5$ A,B,C); $\mathcal{N}(10,1)($ Fig 5 D,E,F) & $(s . \mu M)^{-1}$ \\
\hline$k_{o n}^{t G}$ & activation rate of the tGTPase & 3 (Figs $2,3,4,5$ A,B,C); $\mathcal{N}(30,1)$ (Fig 5 D,E,F) & $(s . \mu M)^{-1}$ \\
\hline$k_{o f f}^{t G}$ & deactivation rate of the tGTPase & 1 (Figs $2,3,4,5$ A,B,C); $\mathcal{N}(10,1)($ Fig 5 D,E,F) & $(s . \mu M)^{-1}$ \\
\hline$k_{o n}^{I}$ & activation rate of tGEF through forward coupling & 0 or 3 (Figs $2,3,4,5$ A,B,C); 0 or $\mathcal{N}(30,1)$ (Fig 5 D,E,F) & $(s . \mu M)^{-1}$ \\
\hline$k_{o n}^{I I}$ & activation rate of mGAP through feedback loop (arrow 2) & 0 or 3 (Figs $2,3,4,5$ A,B,C); 0 or $\mathcal{N}(30,1)$ (Fig 5 D,E,F) & $(s . \mu M)^{-1}$ \\
\hline$\left[t G A P^{*}\right]$ & concentration of active tGAP & 1 (Figs $2,3,4,5$ ) & $\mu M$ \\
\hline$\left[t G_{t o t}\right]$ & Total concentration of tGTPase & 10 (Figs 2,3,4); 5 (Fig 5) & $\mu M$ \\
\hline$\left[t G E F_{t o t}\right]$ & Total concentration of tGEF & 10 (Figs 2,3,4); 5 (Fig 5) & $\mu M$ \\
\hline$[t G E F](0)$ & initial concentration of inactive mGTPase & 5 (Figs $2,3,4,5$ ) & $\mu M$ \\
\hline$\left[t G E F^{*}\right](0)$ & initial concentration of active tGEF & 5 (Figs $2,3,4$ ) or 0 (Fig 5) & $\mu M$ \\
\hline$[m G A P](0)$ & initial concentration of inactive mGAP & $[0,20]$ (Figs 3,4$) ;[0,12]$ (Fig 5) & $\mu M$ \\
\hline$\left[m G A P^{*}\right](0)$ & initial concentration of inactive mGAP & 1 (Figs 2,3,4); [0,12] (Fig 5) & $\mu M$ \\
\hline
\end{tabular}

In Fig.2, we explore the case where the two GTPase switches are coupled through the coupling connection $m G^{*} \rightarrow t G E F$ (Fig.2A). We color the trajectories of the system according to the comparison between the initial conditions $[m G](0)+\left[m G^{*}\right](0)$ and $\left[t G E F^{*}\right](0)$ from the steady state analysis in Section 3.1. For fixed $\left[m G^{*}\right](0)$ and $[t G E F](0)$ values, we consider $[m G](0)$ ranging from 0 to $10 \mu M$ and therefore $[m G](0)+\left[m G^{*}\right](0)$ can be less of higher than $[t G E F](0)$ (blue or red-colored lines and dots). For all simulations, we plot the trajectories of the system until equilibrium is reached. If $[m G](0)+\left[m G^{*}\right](0)<[t G E F](0)$, the system converges to a state where no active mGTPase exists (blue colored trajectories in Figs.2B and 2C). On the other hand, if $[m G](0)+\left[m G^{*}\right](0)>[t G E F](0)$, the system converges a state where the concentration of the active and inactive mGTPase are positive at the final time (red-colored trajectories). To visualize these results in terms of dose-response curves, in Fig.2D we plot the final-state values of $\left[m G_{t o t}\right]$ and $\mathcal{G}^{*}$ (denoted by s.s) as a function of $[m G](0)$. The trajectories in the $\mathcal{T}^{*} \times\left[m G_{t o t}\right]$ plane are shown in Fig.2E. We observe a detail showing that $\mathcal{T}^{*}$ reaches a fixed final value around 0.97 when $[m G](0)+\left[m G^{*}\right](0)>[t G E F](0)$ 
(A)
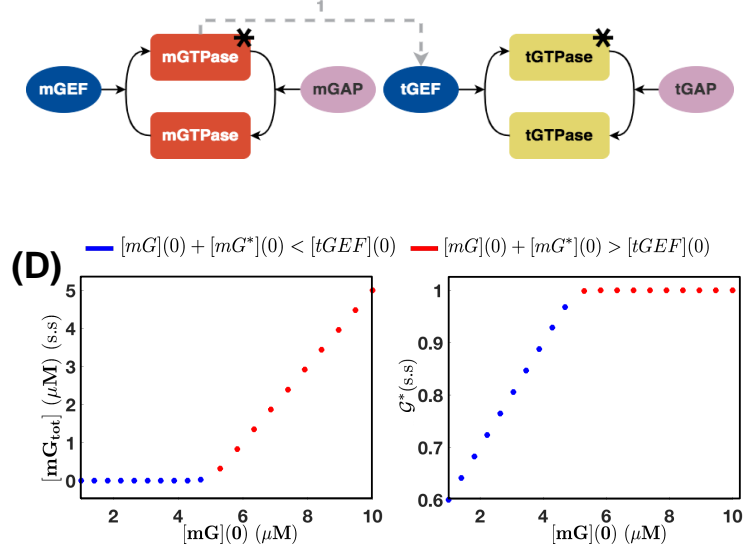

(B)

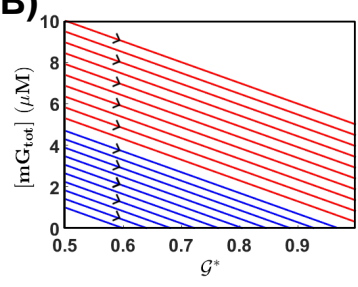

(E)
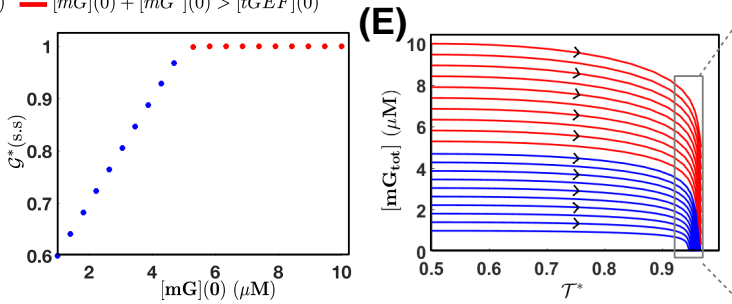

(C)
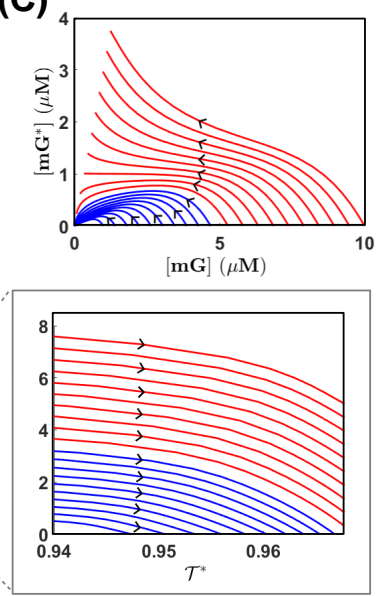

FIGURE 2. Trajectories of the system and steady states (arrow 1) (A) Schematics with the coupled GTPase switches and a coupling connection $m G^{*} \rightarrow t G E F$, represented by arrow 1 . (B) $[m G](0)$ was changed from 0 to $10 \mu M$ and the trajectories of the system were calculated until equilibrium was reached. In the $\mathcal{G}^{*} \times\left[m G_{t o t}\right]$ plane, a linear relationship emerges. The black arrows indicate the direction of time. If $[m G](0)>5 \mu M$, the system converges to a final-state where the concentrations of the active and inactive mGTPase are nonzero. On the other hand, when $[m G](0)<5 \mu M$, the trajectories converge a final-state with no mGTPase exists (blue colored lines). (C) Trajectories of the active $([m G])$ vs inactive mGTPase $([\mathrm{mG}])$ for $[m G](0)$. (D) Dose response curves show the steady states (denoted by s.s) for the total mGTPase concentration and fraction of active tGEF $(\mathcal{G})$ depending on $[m G](0)$ in the two different scenarios. (E) The dynamics in the $\mathcal{T} \times\left[m G_{t o t}\right]$ plane. Parameter values: $\left[m G^{*}\right](0)=0 \mu M, \mathcal{T}^{*}(0)=0.5, \mathcal{G}^{*}(0)=0.5,\left[m G A P^{*}\right]=$ $1 \mu M,\left[m G E F^{*}\right]=1 \mu M, k_{o n}=3(s . \mu M)^{-1}, k_{o f f}=1(s . \mu M)^{-1},\left[t G A P^{*}\right]=$ $1 \mu M,\left[t G E F_{t o t}\right]=10 \mu M,\left[t G_{t o t}\right]=10 \mu M$. Simulation time: $5 s$ for panels B and E, $50 \mathrm{~s}$ for panels C, D, F, and G. Numerical simulations were performed using the solver ode15s in Matlab R2018a. All parameters were arbitrarily chosen only to illustrate the dynamic features of the model.

(see magnified view). We observe that the trajectories converge to steady states that agree with the local stability results from $\S$ Section 3.1. This suggests that the conditions $[m G](0)+\left[m G^{*}\right](0)<[t G E F](0)$ and $[m G](0)+\left[m G^{*}\right](0)>[t G E F](0)$ are not only valid in a neighborhood of the steady states, but also hold for other initial values satisfying those inequalities.

Fig.3 illustrates the dynamics of the system when the feedback loop $t G E F \rightarrow m G A P$ (Arrow 2) is added to the coupling connection (Fig.3A). In Fig.3B, we plot several $\left[t G E F^{*}\right]$ trajectories starting at $\left[t G E F^{*}\right]=5 \mu M$ for different $[m G](0)$ and $[m G A P](0)$ values. The resulting rich variety of curves indicate the sensitivity of the system to these initial conditions. In Fig.3C, different dose-response curves are generated to show 
the steady state $\mathrm{tGEF}^{*}$ values. If $[m G A P](0)=0 \mu M$ (blue and red dots), only the coupling connection affects the system, since mGAP cannot be activated by tGEF*. When $[m G A P](0)=1$ (green squares), a similar steady state profile emerges, with $\left[t G E F^{*}\right]$ s.s increasing for $[m G](0) \leq 5 \mu M$ and remaining constant $[m G](0)>5$. When $[m G A P](0)=8 \mu M,\left[t G E F^{*}\right]$ is zero for $[m G](0)<2 \mu M$ and increases until $[m G](0)<5 \mu M$. For $[m G](0)>5$, the steady state achieves its maximum value slightly above $\left[t G E F^{*}\right]>2$. Finally when $[m G A P](0)=11 \mu M, t G E F^{*}$ becomes fully recruited by mGAP and the $\left[t G E F^{*}\right]$ s.s is zero for all $[m G](0)$ values. In Fig.3D, we scan the space of initial amounts of $\mathrm{mG}$ and mGAP. When $[m G A P](0)>10 \mu M$, the $\left[t G E F^{*}\right]$ s.s is zero, while for $[m G A P](0)<10 \mu M$ is becomes nonzero and dependent of $[m G](0)$. In Figs. $3(\mathrm{E}),(\mathrm{F})$, and $(\mathrm{G})$, we analyze the $\mathrm{tG}^{*}$ concentration values and obtain similar results.

Fig.4 illustrates the dynamics of the system when the feedback loops $t G E F \rightarrow m G A P$ and $t G^{*} \rightarrow m G A P$ are added to the coupling connection (Fig.4A). In Fig.3B, we plot several $\left[t G E F^{*}\right]$ trajectories starting at $\left[t G E F^{*}\right]=5 \mu M$ for different $[m G](0)$ and $[m G A P](0)$ values. In Fig.3C, different dose-response curves are generated to show the steady state $\mathrm{tGEF}^{*}$ values. As in the previous case with only one feedback loop, if $[m G A P](0)=0 \mu M$ (blue and red dots), mGAP cannot be activated by tGEF*. When $[m G A P](0)=1$ (green squares), a similar steady state profile emerges, with $\left[t G E F^{*}\right]$ s.s increasing for $[m G](0) \leq 5 \mu M$ and remaining constant $[m G](0)>5$. When $[m G A P](0)=8$ and $11 \mu M,\left[t G E F^{*}\right]$ increases until $[m G](0)<5 \mu M$. For $[m G](0)>5$, the steady state achieves its maximum value. In Fig.4D, we scan the space of initial amounts of $\mathrm{mG}$ and $\mathrm{mGAP}$ and we observe a more graded response in comparison with Fig.3. In Figs. $4(\mathrm{E}),(\mathrm{F})$, and $(\mathrm{G})$, we analyze the $\mathrm{tG}^{*}$ concentration values and obtain similar results.

In Fig.5, we investigate the space of initial conditions for $\mathrm{mG}^{*}$ and $\mathrm{mGAP}^{*}$ in which the system converges to the different steady states. Fig.5A shows the simplest system where the two GTPase switches are connected by the coupling $m G^{*} \rightarrow t G E F$. Two steady states are obtained depending on the initial amount of $\mathrm{mG}^{*}$. For $\left[m G^{*}\right](0)<$ $[t G E F](0)-\left[m G A P^{*}\right](0)=5 \mu M$, the trajectories converge to steady state 1 with no $\mathrm{mG}$ and $\mathrm{mG}^{*}$ concentrations. On the other hand, for $\left[m G^{*}\right](0)>[t G E F](0)-$ $\left[m G A P^{*}\right](0)=5 \mu M$, then the system achieves the steady state 2 with non zero concentrations of both $\mathrm{m}$ and $\mathrm{t}-\mathrm{GTPase}$. Fig.5B shows the results for the coupling connection, and feedback loops $t G E F \rightarrow m G A P$ (arrows 1+2). In this particular example, the four steady states can be achieved for $\left[m G A P^{*}\right](0)$ and $\left[m G^{*}\right](0)$ ranging from 0 to 12 $\mu M$ and 0 and $10 \mu M$, respectively. In the vertical direction, the initial amount of $\mathrm{mG}^{*}$ governs the transitions from steady states 3 to 4 (lower $\left[m G A P^{*}\right](0)$ ) and 1 to 2 (higher $\left[m G A P^{*}\right](0)$ ). In both steady states 2 and 4 (Eqs. 3.34 and 3.36), the concentrations of mGTPase are nonzero. Therefore, we predict that an increase of initial concentration of $\mathrm{mG}^{*}$ would favor the emergence of these two steady states. In the horizontal direction, 
(A)

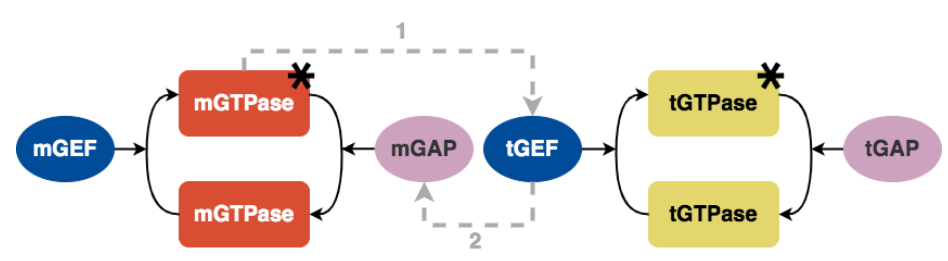

\section{(B)}
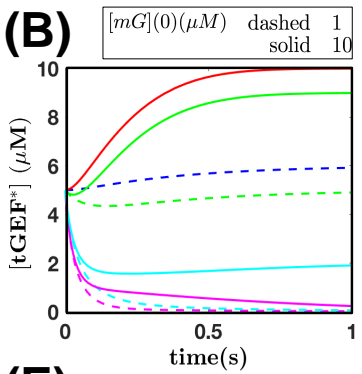

(E)

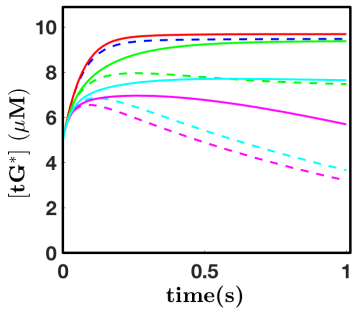

(C)

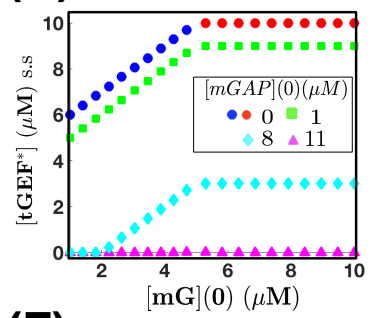

(F)

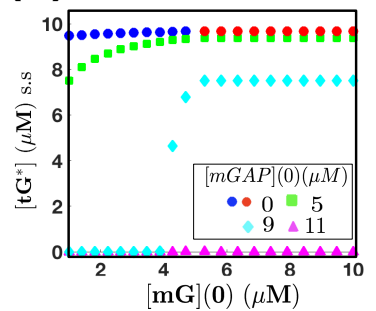

(D)

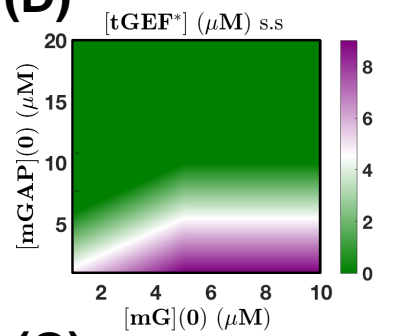

(G)

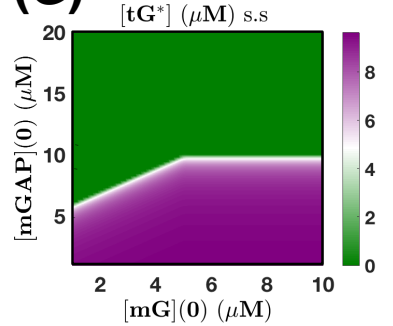

FIGURE 3. Trajectories of the system and steady states (s.s) (arrows 1 and 2). (A) Schematics of the coupled GTPases with the coupling connection $m G^{*} \rightarrow t G E F$ (arrow 1) and the feedback loop $t G E F \rightarrow m G A P$ (arrow 2). (B) $\left[t G E F^{*}\right]$ trajectories for $[m G A P](0)=0,1,8$, and $11 \mu M$. For each $[m G A P](0)$ value, we plot two curves for $[m G](0)=1$ (dashed) and $10 \mu M$ (solid) (C) Dose response curves show $\left[t G E F^{*}\right]$ s.s when $[m G](0)$ ranges from 0 to $10 \mu M$. If $[m G A P](0)=0 \mu M$ (blue and red dots), there will be no mGAP activation and therefore no effects of the feedback. For $[m G A P](0)>0 \mu M$, the feedback becomes effective and generate different $\left[t G E F^{*}\right]$ responses. (D) Colormap for $\left[t G E F^{*}\right]$ s.s concentrations for a range of $[m G](0)$ and $[m G A P](0)$ values. A sharp decrease on $\left[t G E F^{*}\right]$ occurs when $[m G A P](0) \geq 10 \mu M$. When $[m G A P](0)<10 \mu M$, the $\left[t G E F^{*}\right]$ s.s depend on $[m G](0)$. (E) $\left[t G^{*}\right]$ trajectories for $[m G A P](0)=0,5,9$, and $11 \mu M$ and same $[m G](0)$. (F) Dose response curves for $\left[t G^{*}\right]$ s.s depend on $[m G A P](0)$. (G) Colormap for $\left[t G^{*}\right]$ s.s.; lower $t G^{*}$ concentrations result from higher $[m G A P](0)$ values, since tGEF* is recruited for mGAP activation. Parameter values: $k_{o n}=3(s . \mu M s)^{-1}, k_{o} f f=1(s . \mu M)^{-1},\left[m G^{*}\right](0)=0 \mu M$, $\left[t G E F_{\text {tot }}\right](0)=10 \mu M,\left[t G E F^{*}\right](0)=5 \mu M, \mathcal{T}^{*}(0)=0.5,\left[t G_{t o t}\right]=10 \mu M$, $\left[m G A P^{*}\right](0)=1 \mu M,\left[t G A P^{*}\right](0)=1 \mu M,\left[m G E F^{*}\right]=1 \mu M$. Simulation times: $5 s$ (B and $\mathrm{E}$ ) and $50 s$ (C, D, F, and $\mathrm{G})$. Numerical simulations were performed using the solver ode23s in Matlab R2018a. All parameters were arbitrarily chosen only to illustrate the dynamic features of the model.

when the initial amount of mGAP* increases, the available mGAP (inactive) decreases as we set the total mGAP as $12 \mu M$, which reduces the effects of the feedback loops and thus facilitates the emergence of the steady states 1 and 2 where the concentrations of tGTPase are nonzero. 


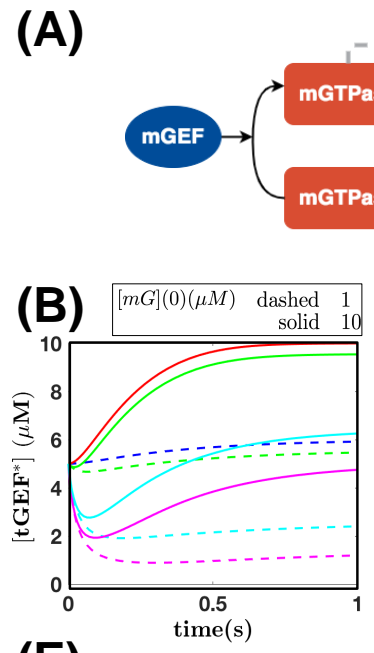

(E)

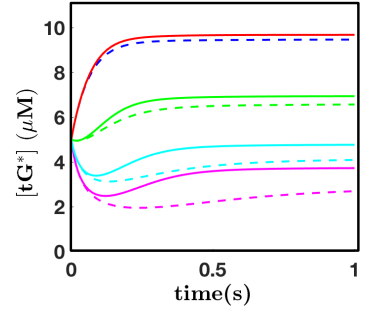

(C)

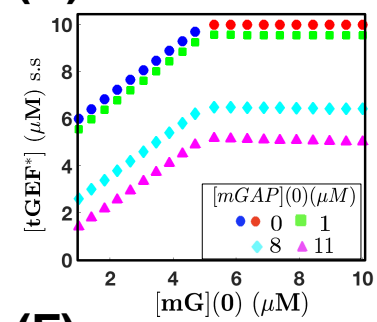

(F)

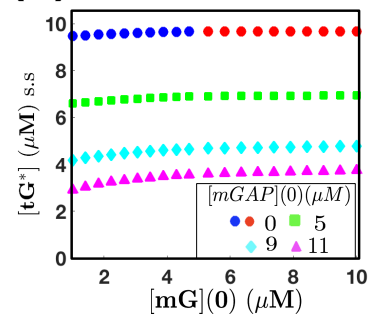

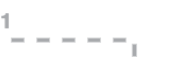

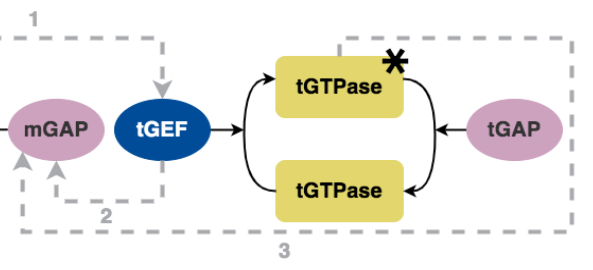

(D)

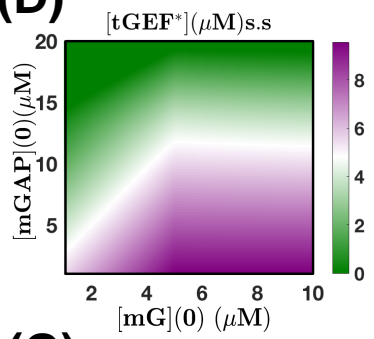

(G)

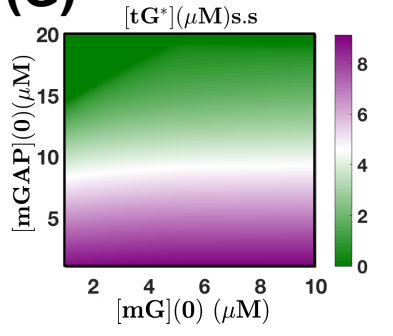

FIGURE 4. Trajectories of the system and steady states (s.s) (arrows 1, 2, and 3). (A) Schematics of the coupled GTPases with the coupling connection $m G^{*} \rightarrow t G E F$ (arrow 1) and the feedback loops $t G E F \rightarrow m G A P$ (arrow 2) and $t G^{*} \rightarrow m G A P$ (arrow 3). (B) $\left[t G E F^{*}\right]$ trajectories for $[m G A P](0)=0,1,8$, and $11 \mu M$. For each $[m G A P](0)$ value, we plot two curves for $[m G](0)=1$ and $10 \mu M$. (C) Dose response curves show $\left[t G E F^{*}\right]$ s.s when $[m G](0)$ ranges from 0 to $10 \mu M$. If $[m G A P](0)=0 \mu M$ (blue and red dots), there will be no mGAP activation and therefore no effects of the feedback loops. For $[m G A P](0)>0 \mu M$, the feedback becomes effective and generate different $\left[t G E F^{*}\right]$ responses. (D) Colormap for $\left[t G E F^{*}\right]$ s.s concentrations for a range of $[m G](0)$ and $[m G A P](0)$ values. A more graded decrease on $\left[t G E F^{*}\right]$ occurs when $[m G A P](0) \geq 10 \mu M$ in comparison with Fig.3D. (E) $\left[t G^{*}\right]$ trajectories for $[m G A P](0)=0,5,9$, and $11 \mu M$ and same $[m G](0)$. (F) Dose response curves for $\left[t G^{*}\right]$ s.s depend on $[m G A P](0)$ and does not change significantly as $[m G](0)$ increases. (G) Colormap for $\left[t G^{*}\right]$ s.s.; lower $\mathrm{tG}^{*}$ concentrations result from higher $[m G A P](0)$ values, since $\mathrm{tGEF}^{*}$ and $\mathrm{tG}^{*}$ are recruited for mGAP activation. Parameter values: $k_{o n}=3(s . \mu M s)^{-1}, k_{o} f f=1(s . \mu M)^{-1},\left[m G^{*}\right](0)=0 \mu M$, $\left[t G E F_{t o t}\right](0)=10 \mu M,\left[t G E F^{*}\right](0)=5 \mu M, \mathcal{T}^{*}(0)=0.5,\left[t G_{t o t}\right]=10 \mu M$, $\left[m G A P^{*}\right](0)=1 \mu M,\left[t G A P^{*}\right](0)=1 \mu M,\left[m G E F^{*}\right]=1 \mu M$. Simulation times: $5 s$ (B and $\mathrm{E}$ ) and $50 s(\mathrm{C}, \mathrm{D}, \mathrm{F}$, and $\mathrm{G})$. Numerical simulations were performed using the solver ode23s in Matlab R2018a. All parameters were arbitrarily chosen only to illustrate the dynamic features of the model.

Fig.5C shows a similar colormap for the system with both feedback loops $t G E F \rightarrow$ $m G A P$ and $t G^{*} \rightarrow m G A P$. It is worth noticing the expansion of the basin of attraction of Families 1 and 2 compared to Fig.5A, while the basin of Families 3 and 4 shrinks. Remarkably, in both Figs. 5B and 5C, there is a critical point (represented by a black 
cross) of intersection of the four basins of attraction. In this case, disturbances in the initial conditions around that intersection point can drive the system to different steady states. Thus, while coupling the two GTPase switches with a forward arrow only gives two possible steady states, the negative feedback afforded by arrows 2 and 3 give rise to a larger range of possibilities. Additionally, the existence of a critical point emerges in the presence of the negative feedback suggesting a rich phase space for this coupled system. Finally, in Figs 5(D-F),we sample the $k_{o n}$ 's from a normal distribution with mean $30(s . \mu M)^{-1}$ and standard deviation $1(s . \mu M)^{-1}$ and new $k_{o f f}$ 's from a normal distribution with mean $10(s . \mu M)^{-1}$ and standard deviation $1(s . \mu M)^{-1}$. We note that the system behavior does not change for changes in kinetic parameters. By doing so, we illustrate how the basins of attraction remain the same, given distinct reaction rates with a different order of magnitude.

\section{DiscusSiON}

GTP-binding proteins (GTPases) regulate crucial aspects of numerous cellular events. Their ability to act as biochemical switches is essential to promote information processing within signaling networks. The two types of GTPases - monomeric $(\mathrm{m})$ and trimeric (t) - have traditionally believed to function independently until recent experimental work revealed that $\mathrm{m}$ - and tGTPases coregulate each other in the Golgi through a functionally coupled circuit [32]. Using a simplified model of ODEs, our analyses have shown that the coupled switch gives rise to steady state configurations that cannot be achieved in systems of isolated GTPase switches. To the best of our knowledge, this is the first modeling effort that has described the stability properties of these coupled GTPase switches.

A major result from our analysis is a systematic characterization of the steady state concentrations of both $\mathrm{m}$ - and tGTPases, as well as their GEFs and GAPs. We show the obtained steady states in all three arrow combinations that were informed by experiments (Table 3). Remarkably, the different steady states show a variety of configurations in which both $\mathrm{m}$ - and tGTPase can be interpreted as having low or high concentration values. We also note that the stability properties of these steady states are independent of the choice of kinetic parameters in this model. We next interpret these different steady states in their biological context.

First and foremost, the coupling of the two switches allows for the emergence of two stable steady states. The first steady state (Eq. 3.7) has zero $\mathrm{mG}$ and $\mathrm{mG}^{*}$ values and finite $\mathrm{t} G$ and $\mathrm{tG}^{*}$ values. This concentration distribution of the species can be interpreted as a scenario in which nearly all the available $\mathrm{mG}$ proteins are activated to $\mathrm{mG}^{*}$, and that nearly all the $\mathrm{mG}^{*}$ species have successfully engaged with the available tGEFs, thereby maximally recruiting tGEF on the Golgi membranes. This steady state emerges when the total amount of $\mathrm{mG}$ protein is less than the concentration of tGEF in cells. Similarly, the steady state 2 will emerge when the total concentration of $\mathrm{mG}$ is greater than $\mathrm{tG}$. In 
(A)

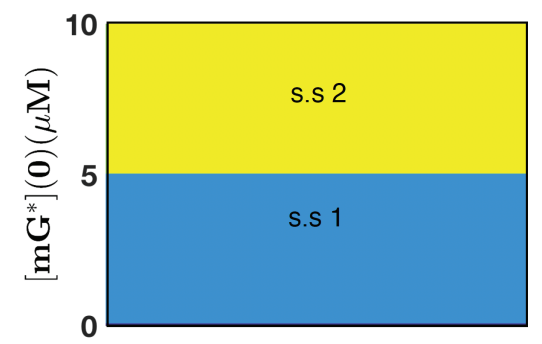

(D)

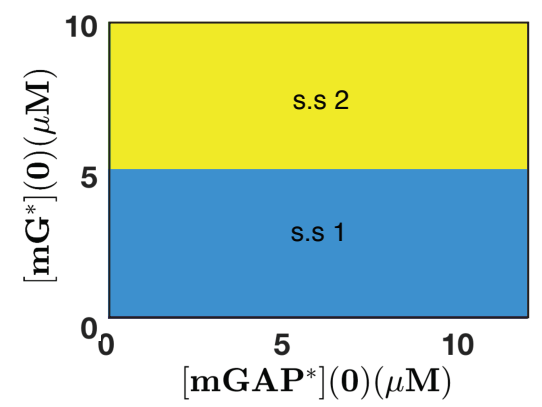

(B)

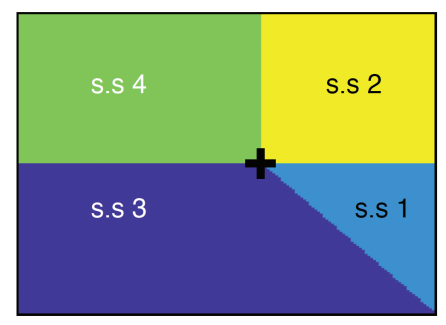

(E)

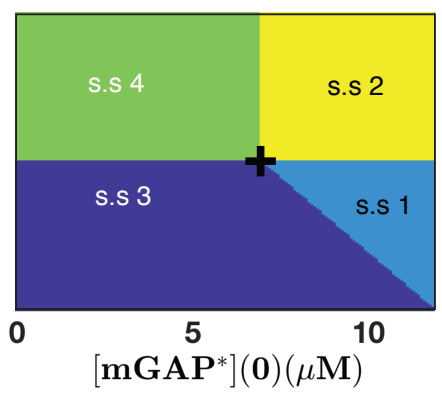

(C)

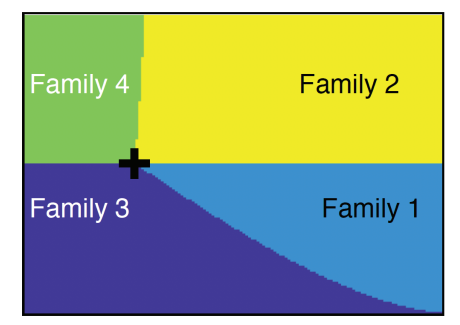

(F)

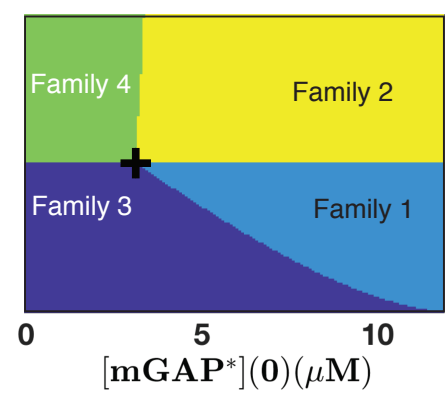

FIGURE 5. Basins of Attraction - dependency on $\left[m G^{*}(0)\right]$ and $\left[m G A P^{*}(0)\right]$ (A) The two steady states of the system with coupling connection (Section 3.1) are only driven by changes in the initial amount of $\mathrm{mG}^{*}$ (B) When the coupling connection and both feedback loops $t G E F \rightarrow m G A P$ are considered, we observe the emergence of four regions (green,yellow, dark blue and light blue colored) corresponding to the four steady states from Section 3.2 (C) A similar result was found when we analyzed the system with the coupling connection and both feedback loops $t G E F \rightarrow m G A P$ and $t G^{*} \rightarrow m G A P$. A black cross indicates a critical point at the intersection of the four basins of attraction. (D), (E), and (F) The basins of attraction remain unaltered when we consider distinct activation/deactivation rates of different orders of magnitude. Parameter values for panels (A), (B), and (C): $k_{o n}^{m G}=k_{o n}^{t G}=3(s . \mu M)^{-1}$, $k_{o n}^{i}=3$ or $0(s . \mu M)^{-1}$ for $i=I, I I$, or $I I I, k_{o f f}^{m G}=k_{o f f}^{t G}=1(s . \mu M)^{-1}$. Reaction rates for panel (D) in $(s . \mu M)^{-1}: k_{o n}^{I}=32.9841, k_{o n}^{I I}=30.8527, k_{o n}^{I I I}=30.4140$, $k_{\text {of }}^{m G}=9.0960, k_{o n}^{m G}=29.4273, k_{o f f}^{t G}=10.4226, k_{o n}^{t G}=29.8986$, Reaction rates for panel (E) in $(s . \mu M)^{-1}: k_{o n}^{I}=31.5946, k_{o n}^{I I}=29.2870, k_{o n}^{I I I}=29.7058$, $k_{o f f}^{m G}=10.1447, k_{o n}^{m G}=31.4250, k_{o f f}^{t G}=10.4106, k_{o n}^{t G}=29.8115$ Reaction rates for panel $(\mathrm{F})$ in $\left.(s . \mu M)^{-1}\right): k_{o n}^{I}=29.8231, k_{o n}^{I I}=28.6578, k_{o n}^{I I I}=31.0219$, $k_{o f f}^{m G}=9.8054, k_{o n}^{m G}=30.1932, k_{o f f}^{t G}=11.2932, k_{o n}^{t G}=29.8454$. Initial conditions: $[m G](0)=0 \mu M,[t G](0)=5 \mu M,\left[t G^{*}\right](0)=0 \mu M,[t G E F](0)=5 \mu M$, $\left[t G E F^{*}\right](0)=0 \mu M,[m G A P](0)=12 \mu M-\left[m G A P^{*}\right](0),\left[t G A P^{*}\right](0)=1 \mu M$, $\left[m G E F^{*}\right](0)=1 \mu M$

this case, the reduced system will converge to steady state 2 where some finite, nonzero distribution of $\mathrm{mG}, \mathrm{mG}^{*}, \mathrm{tG}, \mathrm{tG}^{*}$ are present (Eq. 3.8), given sufficiently close initial and steady state concentration values.

When we couple the connection of the two GTPase switches with the feedback loop $t G E F \rightarrow m G A P$ (arrows 1 and 2 in Fig. $1 \mathrm{C}$, respectively), we obtain four steady states. Steady states 1 and 2 (Eqs. 3.33 and 3.34) are similar to the two steady states obtained 


\begin{tabular}{|c|c|c|c|c|}
\hline Arrows & Name & Interpretation & Required Initial conditions & Conclusion \\
\hline 1 & $\begin{array}{l}\text { Steady-state } 1 \\
\text { Steady-state } 2\end{array}$ & $\begin{array}{l}\text { Low } m G, \mathrm{mG}^{\star} \text {. High } \mathrm{tG}^{\star} \\
\text { High } \mathrm{mG}, \mathrm{mG}^{*} \text {. High } \mathrm{tG}^{*}\end{array}$ & $\begin{array}{l}m G+m^{*} \leq t G E F \\
m G+m G^{*} \geq t G E F\end{array}$ & $\begin{array}{l}\text { Feed-forward allows } m G \text { and } m^{*} \text { to achieve low s.s } \\
\text { concentrations. High s.s concentrations of } m G, m G^{*} \text {, } \\
\text { tG, and } t G^{*} \text { emergeif initial } m G+m G^{*} \text { is initially } \\
\text { higher than tGEF. }\end{array}$ \\
\hline $1+2$ & $\begin{array}{l}\text { Steady-state } 1 \\
\text { Steady-state } 2 \\
\text { Steady-state } 3 \\
\text { Steady-state } 4\end{array}$ & 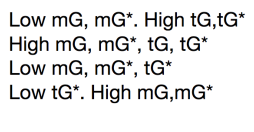 & $\begin{array}{l}m G+\mathrm{mG}^{\star} \leq \mathrm{tGEF} \& \mathrm{mGAP} \leq \mathrm{mG}+\mathrm{mG}^{\star}+\mathrm{tGEF}^{\star} \\
\mathrm{mG}+\mathrm{mG}^{*} \geq \mathrm{tGEF} \& \mathrm{mGAP} \leq \mathrm{tGEF}+\mathrm{tGEF}^{\star} \\
\mathrm{mG}+\mathrm{mG}^{*} \leq \mathrm{tGEF} \& \mathrm{mGAP} \geq \mathrm{mG}+\mathrm{mG}^{*}+\mathrm{tGEF}^{\star} \\
\mathrm{mG}+\mathrm{mG}^{\star} \geq \mathrm{tGEF} \& \mathrm{mGAP} \geq \mathrm{tGEF}+\mathrm{tGEF}^{*}\end{array}$ & $\begin{array}{l}\text { Feed-forward and feedback } t G E F \rightarrow \text { mGAP allow } \\
m G, m G^{*} \text {,and } t G^{*} \text { to achieve low s.s concentrations } \\
\text { simultaneously. High s.s concentrations of } m G \text {, } \\
m G^{*}, t G \text {, and } t^{*} \text { emerge if initials } m G+m G^{*} \text { and } \\
\text { tGEF }+t G E F^{*} \text { are higher than tGEF and } m G A P \text {, resp. }\end{array}$ \\
\hline $1+2+3$ & $\begin{array}{l}\text { Family } 1 \\
\text { Family } 2 \\
\text { Family } 3 \\
\text { Family } 4\end{array}$ & $\begin{array}{l}\text { Low } m G, \mathrm{mG}^{*} \text {. High } \mathrm{tG}, \mathrm{tG} * \\
\text { High } \mathrm{mG}, \mathrm{mG}^{*}, \mathrm{tG} \mathrm{G}^{*} \\
\text { Low } \mathrm{mG}, \mathrm{mG}^{*}, \mathrm{tG} \mathrm{G}^{*} \\
\text { Low } \mathrm{tG} \mathrm{H}^{*}, \mathrm{High} \mathrm{mG}, \mathrm{mG}^{*}\end{array}$ & $\begin{array}{l}m G+m G^{*} \leq t G E F \& \text { } m G A P \leq m G+m G^{*}+t G+t G^{*}+t G E F^{*} \\
m G+m G^{*} \geq t G E F \& \& G A P \leq t G+t G^{*}+t G E F+t G E F^{*} \\
m G+m G^{*} \leq t G E F \\
m G+m G^{*} \geq t G E F\end{array}$ & $\begin{array}{l}\text { Feed-forward, feedback from tGEF to } m G A P \text { and } \\
\text { feedback from } t G^{*} \text { to } m G A P \text { yields four families of } \\
\text { steady-states where the inactive } t G \text { can range within } \\
\text { intervals. }\end{array}$ \\
\hline
\end{tabular}

TABLE. 3. Main results and conclusions from steady state analysis. We performed a steady state analysis of a GTPase coupled circuit that has been observed experimentally. For three biologically relevant combinations among the coupling connection and two feedback loops, we present the steady states and their interpretation. Moreover, we established the required initial conditions for the existence of the steady states. Each connection adds to the richness of the functioning of these coupled GTPase switches.

in Section 3.1, although with different concentration values. On the other hand, steady states 3 and 4 (Eqs. 3.35 and Eqs. 3.36) newly emerge in the system, in which $\mathrm{tG}^{*}$ attains zero concentration. This zero concentration can be interpreted as a scenario in which nearly all the available tGTPase has cycled through the GTP-cycle and is inactivated. The inequalities obtained in Theorem 3.2 for $C_{1}$ and $C_{2}$, allow us to obtain relationships among the initial conditions of the original system (Eqs. $3.10-3.17)$ that are associated with each one of the four steady states. Two conditions guarantee the existence of steady state 1: (1) The total amount of $\mathrm{mG}$ protein must be initially less than the concentration of tGEF and (2) the sum of the concentrations of total $\mathrm{mG}$ protein and tGEF* must be initially higher than the concentration of mGAP. Similar analysis reveals that for steady state 2 (Eq. 3.34), the total amount of $\mathrm{mG}$ protein must be initially higher than the concentration of tGEF and the total amount of tGEF must be initially higher than concentration of mGAP. For steady state 3 (Eq. 3.35), the total amount of $\mathrm{mG}$ protein must be initially less than the concentration of tGEF and the sum of the concentrations of total $\mathrm{mG}$ protein and $\mathrm{tGEF}^{*}$ must be initially less than the concentration of mGAP. For steady state 4 (Eq. 3.36), the total amount of $\mathrm{mG}$ protein must be initially higher than the concentration of tGEF and the total amount of tGEF must be initially less than the concentration of mGAP.

Finally, when the coupled switches have both feedback effects on $m G A P$ through Arrows 2 and 3, we obtain four families of steady states. Interestingly, the Families 1 4 resemble the steady states $1-4$ from Section 3.2. Family 1 has no $m G$ and $\mathrm{mG}^{*}$ at steady state, and both $\mathrm{tG}$ and $\mathrm{tG}^{*}$ have nonzero steady state values (similar to steady state 1). Moreover, Family 2 has both $\mathrm{m}$ - and tGTPases with nonzero steady states (similar to steady state 2). For Family $3, \mathrm{mG}$ and $\mathrm{mG}^{*}$ steady state values are zero, and the tGTPase is fully inactivated (similarly to steady state 3 ). Finally, Family 4 has tGTPase is fully 
inactivated, and both $\mathrm{mG}$ and $\mathrm{mG}^{*}$ have nonzero steady states (similarly to steady state 4). Recalling the definitions of $\tilde{C}_{1}$ and $\tilde{C}_{2}$ and the fact that Eqs. 3.37 and 3.38 hold at all times, including $t=0$, we can infer necessary relationships among the initial conditions for each steady state family.

Thus, our model shows that when the m- and t-GTPase switches are coupled with a simple forward coupling (Arrow 1), there are two steady states. The addition of feedback from the t-GTPase switch to the m-GTPase switch (Arrow 2 alone or Arrows 2 and 3), expands this space to either 4 steady states or 4 families of steady states. We confirmed that all steady states obtained with a coupling connection and feedback loop $t G E F \rightarrow$ $m G A P$ (arrow 1 or arrows $1+2$ in Fig.1C) are locally asymptotically stable. However, when the two feedback loops are considered along with the coupling connection (arrows $1+2+3$ in Fig.1C), the local stability analysis cannot be performed because the steady states are not isolated. Instead, we obtain four one-parameter families that depend on the amount of inactive tGTPase. At this point, further investigation would be needed to determine the behavior of the system near those steady state families. Even as we aim to develop complex models that are refined with iterative experimental validations, we note that our analysis gives insight to different steady states that emerge due to different couplings that may not exist in physiology. Such insights may become meaningful in the context of disease pathogenesis where copy numbers of each player in the network motif may change relative to each other, and do so dynamically (e.g., when responding to stress/stimuli), or disease-driving mutations alter their functions (e.g., activating and inactivating mutations in GTPases, GAPs, or GEFs).

Limitations of this study include a simplified mathematical structure of the model. Despite this simplification, we find a rich phase space for the coupled GTPase switches by analyzing the combination of network connections that have more biological meaning. Future studies could also explore the role of external stimulus, the temporal and spatial organization of these switches. While the current model is likely incomplete, it serves as a stepping stone for future adaptations that can be coupled with experimental measurements [41], including dose-response curves, response times, and noise fluctuations, as done recently in [72]. 


\section{ACKNOWLEDGMENTS}

This work was supported by Air Force Office of Scientific Research (AFOSR) Multidisciplinary University Research Initiative (MURI) grant FA9550-18-1-0051 (to P. Rangamani) and the National Institute of Health (CA100768, CA238042 and AI141630 to P. Ghosh). Lucas M. Stolerman acknowledges support from the National Institute of Health (CA209891). The authors would like to acknowledge Prof. Ali Behzadan (Sacramento State University) for his careful reading and insightful comments for this work.

\section{REFERENCES}

[1] B. Alberts, A. Johnson, J. Lewis, D. Morgan, M. Raff, K. Roberts, and P. Walter, "Molecular biology of the cell. 2015," Garland, New York, pp. 139-194, 2013.

[2] H. E. Hamm, "The many faces of G protein signaling," Journal of Biological Chemistry, vol. 273, no. 2, pp. 669-672, 1998.

[3] H. R. Bourne, D. A. Sanders, and F. McCormick, "The GTPase superfamily: a conserved switch for diverse cell functions," Nature, vol. 348, no. 6297, pp. 125-132, 1990.

[4] A. Lipshtat, G. Jayaraman, J. C. He, and R. Iyengar, "Design of versatile biochemical switches that respond to amplitude, duration, and spatial cues," Proceedings of the National Academy of Sciences, vol. 107, no. 3, pp. 1247-1252, 2010.

[5] K. L. Rossman, C. J. Der, and J. Sondek, "GEF means go: turning on RhoGTPases with guanine nucleotide-exchange factors," Nature reviews Molecular cell biology, vol. 6, no. 2, pp. 167-180, 2005.

[6] J. Wang, Y. Tu, S. Mukhopadhyay, P. Chidiac, G. H. Biddlecome, and E. M. Ross, "GTPase-activating proteins (GAPs) for heterotrimeric G proteins," G Proteins: Techniques of Analysis, pp. 123-151, 1999.

[7] J. L. Bos, H. Rehmann, and A. Wittinghofer, "GEFs and GAPs: critical elements in the control of small G proteins,” Cell, vol. 129, no. 5, pp. 865-877, 2007.

[8] J. Cherfils and M. Zeghouf, "Regulation of small GTPases by GEFs, GAPs, and GDIs," Physiological reviews, vol. 93, no. 1, pp. 269-309, 2013.

[9] D. P. Siderovski and F. S. Willard, "The GAPs, GEFs, and GDIs of heterotrimeric G-protein alpha subunits," International journal of biological sciences, vol. 1, no. 2, p. 51, 2005.

[10] P. Ghosh, P. Rangamani, and I. Kufareva, "The GAPs, GEFs, GDIs and now, GEMs: New kids on the heterotrimeric G protein signaling block," Cell cycle, vol. 16, no. 7, pp. 607-612, 2017.

[11] I. Lopez-Sanchez, Y. Dunkel, Y.-S. Roh, Y. Mittal, S. De Minicis, A. Muranyi, S. Singh, K. Shanmugam, N. Aroonsakool, F. Murray, et al., "GIV/Girdin is a central hub for profibrogenic signalling networks during liver fibrosis," Nature communications, vol. 5, no. 1, pp. 1-18, 2014.

[12] G. S. Ma, N. Aznar, N. Kalogriopoulos, K. K. Midde, I. Lopez-Sanchez, E. Sato, Y. Dunkel, R. L. Gallo, and P. Ghosh, "Therapeutic effects of cell-permeant peptides that activate G proteins downstream of growth factors," Proceedings of the National Academy of Sciences, vol. 112, no. 20, pp. E2602-E2610, 2015.

[13] H. Wang, T. Misaki, V. Taupin, A. Eguchi, P. Ghosh, and M. G. Farquhar, "GIV/girdin links vascular endothelial growth factor signaling to akt survival signaling in podocytes independent of nephrin," Journal of the American Society of Nephrology, vol. 26, no. 2, pp. 314-327, 2015.

[14] A. Hartung, A.-M. Ordelheide, H. Staiger, M. Melzer, H.-U. Häring, and R. Lammers, "The Akt substrate Girdin is a regulator of insulin signaling in myoblast cells," Biochimica et Biophysica Acta (BBA)-Molecular Cell Research, vol. 1833, no. 12, pp. 2803-2811, 2013. 
[15] V. DiGiacomo, M. Maziarz, A. Luebbers, J. M. Norris, P. Laksono, and M. Garcia-Marcos, "Probing the mutational landscape of regulators of G protein signaling proteins in cancer," Science signaling, vol. 13, no. 617, 2020.

[16] D. Hanahan and R. A. Weinberg, "The hallmarks of cancer," cell, vol. 100, no. 1, pp. 57-70, 2000.

[17] G. A. Cardama, N. González, J. Maggio, P. L. Menna, and D. E. Gomez, "RhoGTPases as therapeutic targets in cancer," International journal of oncology, vol. 51, no. 4, pp. 1025-1034, 2017.

[18] W. N. Liu, M. Yan, and A. M. Chan, "A thirty-year quest for a role of R-Ras in cancer: from an oncogene to a multitasking gtpase," Cancer letters, vol. 403, pp. 59-65, 2017.

[19] K. Sriram, K. Moyung, R. Corriden, H. Carter, and P. A. Insel, "GPCRs show widespread differential mrna expression and frequent mutation and copy number variation in solid tumors," PLoS biology, vol. 17, no. 11, p. e3000434, 2019.

[20] V. Wu, H. Yeerna, N. Nohata, J. Chiou, O. Harismendy, F. Raimondi, A. Inoue, R. B. Russell, P. Tamayo, and J. S. Gutkind, "Illuminating the Onco-GPCRome: Novel G protein-coupled receptordriven oncocrine networks and targets for cancer immunotherapy," Journal of Biological Chemistry, vol. 294, no. 29, pp. 11062-11086, 2019.

[21] M. O’hayre, J. Vázquez-Prado, I. Kufareva, E. W. Stawiski, T. M. Handel, S. Seshagiri, and J. S. Gutkind, "The emerging mutational landscape of G proteins and G-protein-coupled receptors in cancer," Nature Reviews Cancer, vol. 13, no. 6, pp. 412-424, 2013.

[22] M. Garcia-Marcos, P. Ghosh, and M. G. Farquhar, "GIV is a nonreceptor gef for Goi with a unique motif that regulates Akt signaling," Proceedings of the National Academy of Sciences, vol. 106, no. 9, pp. 3178-3183, 2009.

[23] P. Ghosh, "Heterotrimeric G proteins as emerging targets for network based therapy in cancer: End of a long futile campaign striking heads of a Hydra," Aging (Albany NY), vol. 7, no. 7, p. 469, 2015.

[24] M. M. Papasergi, B. R. Patel, and G. G. Tall, "The G protein $\alpha$ chaperone Ric-8 as a potential therapeutic target," Molecular pharmacology, vol. 87, no. 1, pp. 52-63, 2015.

[25] E. Evers, G. Zondag, A. Malliri, L. Price, J.-P. Ten Klooster, R. Van Der Kammen, and J. Collard, "Rho family proteins in cell adhesion and cell migration," European journal of cancer, vol. 36, no. 10, pp. 1269-1274, 2000.

[26] S. Etienne-Manneville and A. Hall, "RhoGTPases in cell biology," Nature, vol. 420, no. 6916, pp. 629-635, 2002.

[27] Y. Takai, T. Sasaki, and T. Matozaki, "Small GTP-binding proteins," Physiological reviews, vol. 81, no. 1, pp. 153-208, 2001.

[28] A. G. Gilman, "G proteins: transducers of receptor-generated signals," Annual review of biochemistry, vol. 56, no. 1, pp. 615-649, 1987.

[29] F. A. Barr, A. Leyte, and W. B. Huttner, “Trimeric G proteins and vesicle formation," Trends in cell biology, vol. 2, no. 4, pp. 91-94, 1992.

[30] J. L. Stow, J. B. De Almeida, N. Narula, E. J. Holtzman, L. Ercolani, and D. A. Ausiello, “A heterotrimeric $\mathrm{G}$ protein, $\mathrm{G}$ alpha i-3, on Golgi membranes regulates the secretion of a heparan sulfate proteoglycan in LLC-PK1 epithelial cells.," The Journal of Cell Biology, vol. 114, no. 6, pp. 11131124, 1991.

[31] J. Cancino and A. Luini, "Signaling circuits on the Golgi complex," Traffic, vol. 14, no. 2, pp. 121134, 2013.

[32] I.-C. Lo, V. Gupta, K. K. Midde, V. Taupin, I. Lopez-Sanchez, I. Kufareva, R. Abagyan, P. A. Randazzo, M. G. Farquhar, and P. Ghosh, "Activation of Goi at the Golgi by GIV/Girdin imposes finiteness in Arf1 signaling," Developmental cell, vol. 33, no. 2, pp. 189-203, 2015.

[33] J. G. Donaldson, A. Honda, and R. Weigert, "Multiple activities for Arf1 at the Golgi complex," Biochimica et Biophysica Acta (BBA)-Molecular Cell Research, vol. 1744, no. 3, pp. 364-373, 2005. 
bioRxiv preprint doi: https://doi.org/10.1101/2020.08.31.276311; this version posted December 15,2020 . The copyright holder for this preprint (which was not certified by peer review) is the author/funder, who has granted bioRxiv a license to display the preprint in perpetuity. It is made available under aCC-BY-ND 4.0 International license.

[34] U. Rein, U. Andag, R. Duden, H. D. Schmitt, and A. Spang, "ARF-GAP-mediated interaction between the ER-golgi v-SNAREs and the COPI coat," The Journal of cell biology, vol. 157, no. 3, pp. 395-404, 2002.

[35] S. Chantalat, R. Courbeyrette, F. Senic-Matuglia, C. L. Jackson, B. Goud, and A. Peyroche, "A novel Golgi membrane protein is a partner of the ARF exchange factors Gea1p and Gea2p," Molecular biology of the cell, vol. 14, no. 6, pp. 2357-2371, 2003.

[36] U. Alon, An introduction to systems biology: design principles of biological circuits. CRC press, 2019.

[37] J. M. Bower and H. Bolouri, Computational modeling of genetic and biochemical networks. MIT press, 2001.

[38] N. J. Eungdamrong and R. Iyengar, "Modeling cell signaling networks," Biology of the Cell, vol. 96, no. 5, pp. 355-362, 2004.

[39] A. E. Cowan, I. I. Moraru, J. C. Schaff, B. M. Slepchenko, and L. M. Loew, "Spatial modeling of cell signaling networks," in Methods in cell biology, vol. 110, pp. 195-221, Elsevier, 2012.

[40] M. K. Morris, J. Saez-Rodriguez, P. K. Sorger, and D. A. Lauffenburger, "Logic-based models for the analysis of cell signaling networks," Biochemistry, vol. 49, no. 15, pp. 3216-3224, 2010.

[41] M. Getz, L. Swanson, D. Sahoo, P. Ghosh, and P. Rangamani, "A predictive computational model reveals that GIV/girdin serves as a tunable valve for EGFR-stimulated cyclic AMP signals," Molecular biology of the cell, vol. 30, no. 13, pp. 1621-1633, 2019.

[42] R. Milo, S. Shen-Orr, S. Itzkovitz, N. Kashtan, D. Chklovskii, and U. Alon, "Network motifs: simple building blocks of complex networks," Science, vol. 298, no. 5594, pp. 824-827, 2002.

[43] A. Goldbeter and D. E. Koshland, "An amplified sensitivity arising from covalent modification in biological systems," Proceedings of the National Academy of Sciences, vol. 78, no. 11, pp. 6840$6844,1981$.

[44] J. E. Ferrell Jr, "Self-perpetuating states in signal transduction: positive feedback, double-negative feedback and bistability," Current opinion in cell biology, vol. 14, no. 2, pp. 140-148, 2002.

[45] N. T. Ingolia and A. W. Murray, "Positive-feedback loops as a flexible biological module," Current biology, vol. 17, no. 8, pp. 668-677, 2007.

[46] J. E. Ferrell Jr and W. Xiong, "Bistability in cell signaling: How to make continuous processes discontinuous, and reversible processes irreversible," Chaos: An Interdisciplinary Journal of Nonlinear Science, vol. 11, no. 1, pp. 227-236, 2001.

[47] U. Alon, "Network motifs: theory and experimental approaches," Nature Reviews Genetics, vol. 8, no. 6, pp. 450-461, 2007.

[48] S. R. Neves, P. T. Ram, and R. Iyengar, "G Protein Pathways,” vol. 296, no. 5573, p. 1636.

[49] S. S. Shen-Orr, R. Milo, S. Mangan, and U. Alon, "Network motifs in the transcriptional regulation network of escherichia coli," Nature genetics, vol. 31, no. 1, pp. 64-68, 2002.

[50] U. S. Bhalla and R. Iyengar, "Emergent properties of networks of biological signaling pathways," Science, vol. 283, no. 5400, pp. 381-387, 1999.

[51] U. S. Bhalla and R. Iyengar, "Robustness of the bistable behavior of a biological signaling feedback loop," Chaos: An Interdisciplinary Journal of Nonlinear Science, vol. 11, no. 1, pp. 221-226, 2001.

[52] E. A. Logsdon, S. D. Finley, A. S. Popel, and F. M. Gabhann, "A systems biology view of blood vessel growth and remodelling," Journal of cellular and molecular medicine, vol. 18, no. 8, pp. 1491-1508, 2014.

[53] S. D. Finley, L. J. Broadbelt, and V. Hatzimanikatis, "Computational framework for predictive biodegradation,” Biotechnology and bioengineering, vol. 104, no. 6, pp. 1086-1097, 2009. 
[54] S. D. Finley and A. S. Popel, "Effect of tumor microenvironment on tumor VEGF during anti-VEGF treatment: systems biology predictions," JNCI: Journal of the National Cancer Institute, vol. 105, no. 11, pp. 802-811, 2013.

[55] P. Yen, S. D. Finley, M. O. Engel-Stefanini, and A. S. Popel, "A two-compartment model of VEGF distribution in the mouse," PloS one, vol. 6, no. 11, p. e27514, 2011.

[56] G. Hornung and N. Barkai, "Noise propagation and signaling sensitivity in biological networks: a role for positive feedback," PLoS Comput Biol, vol. 4, no. 1, p. e8, 2008.

[57] S. Hooshangi, S. Thiberge, and R. Weiss, "Ultrasensitivity and noise propagation in a synthetic transcriptional cascade," Proceedings of the National Academy of Sciences, vol. 102, no. 10, pp. 3581$3586,2005$.

[58] T. Shibata and K. Fujimoto, "Noisy signal amplification in ultrasensitive signal transduction," Proceedings of the National Academy of Sciences, vol. 102, no. 2, pp. 331-336, 2005.

[59] J. M. Pedraza and A. van Oudenaarden, "Noise propagation in gene networks," Science, vol. 307, no. 5717, pp. 1965-1969, 2005.

[60] L. Qiao, W. Zhao, C. Tang, Q. Nie, and L. Zhang, "Network topologies that can achieve dual function of adaptation and noise attenuation," Cell systems, vol. 9, no. 3, pp. 271-285, 2019.

[61] S. H. Strogatz, Nonlinear Dynamics And Chaos: With Applications To Physics, Biology, Chemistry And Engineering. Westview Press, first edition edition ed., 1994.

[62] L. Perko, Differential equations and dynamical systems, vol. 7. Springer Science \& Business Media, 2013.

[63] C. Jamora, P. A. Takizawa, R. F. Zaarour, C. Denesvre, D. J. Faulkner, and V. Malhotra, "Regulation of Golgi structure through heterotrimeric G proteins," Cell, vol. 91, no. 5, pp. 617-626, 1997.

[64] J. L. Stow and K. Heimann, "Vesicle budding on Golgi membranes: regulation by G proteins and myosin motors," Biochimica et Biophysica Acta (BBA)-Molecular Cell Research, vol. 1404, no. 1-2, pp. 161-171, 1998.

[65] J. Stow, "Regulation of vesicle trafficking by G proteins," Curr. Opin. Nephrol. Hypertens, vol. 4, pp. 421-425, 1995.

[66] D. T. Gillespie, "Deterministic limit of stochastic chemical kinetics," The Journal of Physical Chemistry B, vol. 113, no. 6, pp. 1640-1644, 2009.

[67] S. K. Hahl and A. Kremling, "A comparison of deterministic and stochastic modeling approaches for biochemical reaction systems: On fixed points, means, and modes," Frontiers in genetics, vol. 7, p. 157, 2016.

[68] J.-P. Changeux, J. Thiéry, Y. Tung, and C. Kittel, "On the cooperativity of biological membranes," Proceedings of the National Academy of Sciences of the United States of America, vol. 57, no. 2, p. 335, 1967.

[69] I. Famili and B. O. Palsson, "The convex basis of the left null space of the stoichiometric matrix leads to the definition of metabolically meaningful pools," Biophysical journal, vol. 85, no. 1, pp. 16-26, 2003.

[70] P. Rangamani and L. Sirovich, "Survival and apoptotic pathways initiated by TNF- $\alpha$ : Modeling and predictions," Biotechnology and bioengineering, vol. 97, no. 5, pp. 1216-1229, 2007.

[71] H. E. Nusse and J. A. Yorke, Dynamics: numerical explorations: accompanying computer program dynamics, vol. 101. Springer, 2012.

[72] K. R. Ghusinga, R. D. Jones, A. M. Jones, and T. C. Elston, "Molecular switch architecture drives response properties," bioRxiv, 2020. 
Appendix A. Proof of Proposition 3.1

We must find nonnegative $\widehat{[m G]}, \widehat{\left[m G^{*}\right]}, \widehat{\mathcal{G}^{*}}$, and $\widehat{\mathcal{T}^{*}}$ satisfying the following system:

$$
\begin{aligned}
& \widehat{[m G]}-\rho_{o f f}^{m G}\left[m G A P^{*}\right] \widehat{\left[m G^{*}\right]}-\rho_{o n}^{I}\left[t G E F_{t o t}\right]\left(1-\widehat{\mathcal{G}^{*}}\right) \widehat{\left[m G^{*}\right]}=0 \\
& \rho_{o n}^{t G}\left[t G E F_{t o t}\right] \widehat{\mathcal{G}^{*}}\left(1-\widehat{\mathcal{T}^{*}}\right)-\rho_{o f f}^{t G} \widehat{\mathcal{T}^{*}}=0 \\
& \left(1-\widehat{\mathcal{G}^{*}}\right) \widehat{\left[m G^{*}\right]}=0 \\
& \widehat{[m G]}+\widehat{\left[m G^{*}\right]}+\left[t G E F_{t o t}\right] \widehat{\mathcal{G}^{*}}=C
\end{aligned}
$$

From Eq. A.3, we must have $\widehat{\left[m G^{*}\right]}=0$ or $\widehat{\mathcal{G}^{*}}=1$. Thus we divide the steady state analysis in two cases.

Case 1: $\widehat{\left[m G^{*}\right]}=0$.

From Eq. A.1 we must have $\widehat{[m G]}=0$ and from Eq. A.4, we obtain $\widehat{\mathcal{G}^{*}}=\frac{C}{\left[t G E F_{t o t}\right]}$. Since $\widehat{\mathcal{G}^{*}} \leq 1$ by definition, we conclude that

$$
C \leq\left[t G E F_{t o t}\right]
$$

Eq. A.5 is also sufficient for $\widehat{\left[m G^{*}\right]}=0$. Otherwise, if $C \leq\left[t G E F_{t o t}\right]$ and $\widehat{\left[m G^{*}\right]}>0$, then $\widehat{\mathcal{G}^{*}}=1$ (Eq. A.3) and from Eq. A.4, we would conclude that $\widehat{[m G]}+\widehat{\left[m G^{*}\right]} \leq 0$, which is impossible.

Finally, by substituting $\widehat{\mathcal{G}^{*}}$ in Eq. A.2, we obtain $\widehat{\mathcal{T}^{*}}=\frac{1}{1+\frac{\rho_{\text {off }}^{t G}}{\rho_{o n}^{t G} C}}$ and therefore the steady state is given by

$$
\left(\widehat{[m G]}, \widehat{\left[m G^{*}\right]}, \widehat{\mathcal{T}^{*}}, \widehat{\mathcal{G}^{*}}\right)=\left(0,0, \frac{1}{1+\frac{\rho_{o f f}^{t G}}{\rho_{o n}^{t G} C}}, \frac{C}{\left[t G E F_{t o t}\right]}\right)
$$

Case $2: \widehat{\mathcal{G}^{*}}=1$

In this case, $\widehat{\left[m G^{*}\right]} \geq 0$ and from Eqs. A.1 and A.4,we obtain

$$
\widehat{\left[m G^{*}\right]}=\frac{C-\left[t G E F_{t o t}\right]}{1+\rho_{o f f}^{m G}\left[m G A P^{*}\right]}
$$

and

$$
\widehat{[m G]}=\frac{\rho_{o f f}^{m G}\left[m G A P^{*}\right]}{1+\rho_{o f f}^{m G}\left[m G A P^{*}\right]}\left(C-\left[t G E F_{t o t}\right]\right)
$$

In this case, since the steady state has to be nonnegative, we must have

$$
C \geq\left[t G E F_{t o t}\right]
$$


which is also sufficient for $\widehat{\mathcal{G}^{*}}=1$. Otherwise if $C \geq\left[t G E F_{\text {tot }}\right]$ and $\widehat{\mathcal{G}^{*}}<1$, then $\widehat{\left[m G^{*}\right]}=\widehat{[m G]}=0$ (Eqs. A.1 and A.3) and, from Eq. A.4, we would have

$$
C=\widehat{[m G]}+\widehat{\left[m G^{*}\right]}+\left[t G E F_{t o t}\right] \widehat{\mathcal{G}^{*}}<\left[t G E F_{t o t}\right]
$$

which is impossible.

Finally, by substituting $\widehat{\mathcal{G}^{*}}=1$ in Eq. A.2, we obtain

$$
\rho_{o n}^{t G}\left[t G E F_{t o t}\right]\left(1-\widehat{\mathcal{T}^{*}}\right)-\rho_{o f f}^{t G} \widehat{\mathcal{T}^{*}}=0
$$

which gives $\widehat{\mathcal{T}^{*}}=\frac{\rho_{\text {on }}^{t G}\left[t G E F_{t o t}\right]}{\rho_{o n}^{t G}\left[t G E F_{t o t}\right]+\rho_{o f f}^{t G}}$ and therefore

$$
\begin{aligned}
\left(\widehat{[m G]}, \widehat{\left[m G^{*}\right]}, \widehat{\mathcal{T}^{*}}, \widehat{\mathcal{G}^{*}}\right)= & \left(\frac{\rho_{o f f}^{m G}\left[m G A P^{*}\right]}{1+\rho_{o f f}^{m G}\left[m G A P^{*}\right]}\left(C-\left[t G E F_{t o t}\right]\right),\right. \\
& \frac{C-\left[t G E F_{t o t}\right]}{1+\rho_{o f f}^{m G}\left[m G A P^{*}\right]}, \\
& \left.\frac{\rho_{o n}^{t G}\left[t G E F_{t o t}\right]}{\rho_{o n}^{t G}\left[t G E F_{t o t}\right]+\rho_{o f f}^{t G}}, 1\right) .
\end{aligned}
$$




\section{Appendix B. Proof of Theorem 3.2}

We begin our proof by computing the steady states of the system, which are solutions of the algebraic system given by Eqs. 3.27-3.32. We also establish necessary and sufficient conditions involving the parameters $C_{1}, C_{2}$, and $\left[m G A P_{t o t}\right]$ for the existence of each steady state. We then compute the Jacobian matrix of the system and determine the local stability of the steady state based on the classical linearization procedure [61].

Steady states. We divide our analysis into four different cases that emerge from the preliminary inspection of the system given by Eqs. 3.27-3.32.

Case 1: $\widehat{\left[m G^{*}\right]}=0$ and $\left[\widehat{m G A P}^{*}\right]=\left[m G A P_{t o t}\right]$.

From Eq. 3.27, we have $\widehat{[m G]}=0$ and from Eq. 3.31, [t $\left.\widehat{t E E F^{*}}\right]=C_{1}-\left[m G A P_{t o t}\right]$. Thus $C_{1} \geq\left[m G A P_{t o t}\right]$ since the steady state must be nonnegative. Now Eq. 3.32 gives $[\widehat{t G E F}]=C_{2}-C_{1}$ and that implies $C_{2} \geq C_{1}$.

Finally, Eq. 3.28 yields

$$
\rho_{o n}^{t G}\left(C_{1}-\left[m G A P_{t o t}\right]\right)\left(1-\widehat{\mathcal{T}^{*}}\right)-\rho_{o f f}^{t G} \widehat{\mathcal{T}^{*}}=0
$$

and hence

$$
\widehat{\mathcal{T}^{*}}=\frac{\rho_{o n}^{t G}\left(C_{1}-\left[m G A P_{t o t}\right]\right)}{\rho_{o n}^{t G}\left(C_{1}-\left[m G A P_{t o t}\right]\right)+\rho_{o f f}^{t G}}
$$

The steady state is therefore given by

$$
\widehat{\mathbf{x}}=\left(0,0, \frac{\rho_{o n}^{t G}\left(C_{1}-\left[m G A P_{t o t}\right]\right)}{\rho_{o n}^{t G}\left(C_{1}-\left[m G A P_{t o t}\right]\right)+\rho_{o f f}^{t G}}, C_{2}-C_{1}, C_{1}-\left[m G A P_{t o t}\right],\left[m G A P_{t o t}\right]\right) .
$$

We now observe that the two parameter relations

$$
C_{1} \geq\left[m G A P_{t o t}\right] \quad \text { and } \quad C_{2} \geq C_{1}
$$

are sufficient for $\widehat{\left[m G^{*}\right]}=0$ and $\left[\widehat{m G A P}^{*}\right]=\left[m G A P_{t o t}\right]$. First, we observe that if $C_{2} \geq C_{1}$ then $\widehat{\left[m G^{*}\right]}=0$. In fact, by subtracting 3.31 from Eq. 3.32, we obtain

$$
[\widehat{t G E F}]-\widehat{[m G]}+\widehat{\left[m G^{*}\right]}=C_{2}-C_{1} \geq 0
$$

and hence $[\widehat{t G E F}] \geq \widehat{[m G]}+\widehat{\left[m G^{*}\right]}$. On the other hand, from Eq. 3.29, we must have $[\widehat{t G E F}]=0$ or $\widehat{\left[m G^{*}\right]}=0$. Thus if $[\widehat{t G E F}]=0$ then $\widehat{[m G]}+\widehat{\left[m G^{*}\right]} \leq 0$ and hence the nonnegativeness of the steady state implies $\widehat{[m G]}=\widehat{\left[m G^{*}\right]}=0$. Now, Eq. 3.31 gives $\left[{\widehat{t G E F^{*}}}^{*}=C_{1}-\left[\widehat{m G A P}^{*}\right]\right.$ and from Eq. 3.30, we must have $\left[\widehat{m G A P}^{*}\right]=\left[m G A P_{t o t}\right]$ or $\left[t \widehat{t E F}^{*}\right]=0$. If $\left[t \widehat{G E F}^{*}\right]=0$, then $\left[{\widehat{m G A P^{*}}}^{*}=C_{1} \geq\left[m G A P_{t o t}\right]\right.$ and hence $\left[\widehat{m G A P}^{*}\right]=\left[m G A P_{t o t}\right]$. Therefore, we have shown that Eq. B.1 imply $\widehat{\left[m G^{*}\right]}=0$ and $\left[\widehat{m G A P}^{*}\right]=\left[m G A P_{t o t}\right]$. Consequently, the steady state in this case must be given by Eq. 3.33.

Case 2: $[\widehat{t G E F}]=0$ and $\left[\widehat{m G A P}^{*}\right]=\left[m G A P_{t o t}\right]$ 
From Eq. 3.32, $\left[t \widehat{t G E F}^{*}\right]=C_{2}-\left[m G A P_{t o t}\right]$ and hence $\left[m G A P_{t o t}\right] \leq C_{2}$. From Eq. 3.31, we must have $\widehat{[m G]}+\widehat{\left[m G^{*}\right]}=C_{1}-C_{2}$ and that implies $C_{1} \geq C_{2}$. Now, Eq. 3.27 gives

$$
\left(C_{1}-C_{2}-\widehat{\left[m G^{*}\right]}\right)=\rho_{o f f}^{m G}\left[m G A P_{t o t}\right] \widehat{\left[m G^{*}\right]}
$$

and therefore

From Eq. 3.28, we must have

$$
\rho_{\text {on }}^{t G}\left(C_{2}-\left[m G A P_{t o t}\right]\right)\left(1-\widehat{\mathcal{T}^{*}}\right)-\rho_{\text {off }}^{t G} \widehat{\mathcal{T}^{*}}=0
$$

from which we obtain

$$
\widehat{\mathcal{T}^{*}}=\frac{\rho_{o n}^{t G}\left(C_{2}-\left[m G A P_{t o t}\right]\right)}{\rho_{o n}^{t G}\left(C_{2}-\left[m G A P_{t o t}\right]\right)+\rho_{o f f}^{t G}}
$$

and therefore the steady state is given by

$$
\begin{aligned}
\widehat{\mathbf{x}}= & \left(\frac{\rho_{o f f}^{m G}\left[m G A P_{t o t}\right]}{1+\rho_{o f f}^{m G}\left[m G A P_{t o t}\right]}\left(C_{1}-C_{2}\right), \frac{\left(C_{1}-C_{2}\right)}{1+\rho_{o f f}^{m G}\left[m G A P_{t o t}\right]},\right. \\
& \left.\frac{\rho_{o n}^{t G}\left(C_{2}-\left[m G A P_{t o t}\right]\right)}{\left(C_{2}-\left[m G A P_{t o t}\right]\right)+\rho_{o f f}^{t G}}, 0, C_{2}-\left[m G A P_{t o t}\right],\left[m G A P_{t o t}\right]\right)
\end{aligned}
$$

We now observe that the two parameter relations

$$
C_{2} \geq\left[m G A P_{t o t}\right] \quad \text { and } \quad C_{1} \geq C_{2}
$$

are sufficient for $[\widehat{t G E F}]=0$ and $\left[\widehat{m G A P}^{*}\right]=\left[m G A P_{t o t}\right]$.

In fact, if $C_{1} \geq C_{2}$ then $[\widehat{t G E F}]=0$ from the same argument as in Case 1. Now, Eq. 3.32 gives $\left[t \widehat{t E F}^{*}\right]=C_{2}-\left[\widehat{m G A P}^{*}\right]$ and from Eq. 3.30, we must have $\left[\widehat{m G A P}^{*}\right]=$ $\left[m G A P_{t o t}\right]$ or $\left[t \widehat{t E F}^{*}\right]=0$. If $\left[\widehat{t G E F}^{*}\right]=0$ then $\left[\widehat{m G A P^{*}}\right]=C_{2} \geq\left[m G A P_{t o t}\right]$ (from Eq. B.2) and thus $\left[\widehat{\left.m G A P^{*}\right]}=\left[m G A P_{t o t}\right]\right.$. Therefore, we have shown that Eq. B.2 imply $[\widehat{t G E F}]=0$ and $\left[\widehat{m G A P}^{*}\right]=\left[m G A P_{t o t}\right]$. Consequently, the steady state in this case must be given by Eq. 3.34.

Case 3: $\widehat{\left[m G^{*}\right]}=0$ and $\left[\widehat{\left.t G E F^{*}\right]}=0\right.$.

From Eq. 3.27, we have $\widehat{[m G]}=0$ and from Eq. 3.28, we also get $\widehat{\mathcal{T}^{*}}=0$ since $\rho_{\text {off }}^{t G}>0$. Now, Eq. 3.31 gives $\left[\widehat{m G A P}^{*}\right]=C_{1}$ and thus we must have $C_{1} \leq\left[m G A P_{t o t}\right]$. Moreover, Eq. 3.32 results in $[\widehat{t G E F}]=C_{2}-C_{1}$ and since all steady states must be nonnegative, we obtain $C_{2} \geq C_{1}$. In this case, the steady state is given by

$$
\widehat{\mathbf{x}}=\left(0,0,0, C_{2}-C_{1}, 0, C_{1}\right)
$$

We now observe that the two parameter relations

$$
C_{1} \leq\left[m G A P_{t o t}\right] \quad \text { and } \quad C_{2} \geq C_{1}
$$


are sufficient for $\widehat{\left[m G^{*}\right]}=0$ and $\left[\widehat{\left.t G E F^{*}\right]}=0\right.$. In fact, $C_{2} \geq C_{1}$ implies $\widehat{\left[m G^{*}\right]}=0$ from the same argument as in Case 1.

Now, Eq. 3.31 gives $\left[\widehat{t G E F}^{*}\right]=C_{1}-\left[\widehat{m G A P}^{*}\right]$ and from Eq. 3.30, we must have $\left[{\widehat{t G E F^{*}}}^{*}=0\right.$ or $\left[\widehat{m G A P}^{*}\right]=\left[m G A P_{t o t}\right]$. If $\left[\widehat{m G A P}^{*}\right]=\left[m G A P_{t o t}\right]$, then $\left[{\widehat{t G E F^{*}}}^{*}=\right.$ $C_{1}-\left[m G A P_{t o t}\right] \leq 0$ (from Eq. B.4) and thus $\left[\widehat{t G E F}^{*}\right]=0$. Therefore, we have shown that Eq. B.4 imply $\widehat{\left[m G^{*}\right]}=0$ and $\left[\widehat{\left.t G E F^{*}\right]}=0\right.$. Consequently, the steady state in this case must be given by Eq. 3.35 .

Case $4:[\widehat{t G E F}]=0$ and $\left[\widehat{t G E F}^{*}\right]=0$

From Eq. 3.32, we obtain $\left[\widehat{m G A P}^{*}\right]=C_{2}$ and hence $C_{2} \leq\left[m G A P_{\text {tot }}\right]$. From Eq. 3.31, we have $\widehat{[m G]}+\widehat{\left[m G^{*}\right]}=C_{1}-C_{2}$ and that implies $C_{1} \geq C_{2}$ since the concentrations at steady state must be nonnegative. Eq. 3.27 then gives

from which we obtain

$$
\widehat{\left[m G^{*}\right]}=\frac{1}{1+\rho_{o f f}^{m G} C_{2}}\left(C_{1}-C_{2}\right) \text { and } \widehat{[m G]}=\frac{\rho_{o f f}^{m G} C_{2}}{1+\rho_{o f f}^{m G} C_{2}}\left(C_{1}-C_{2}\right) .
$$

From Eq. 3.28, we have $\widehat{\mathcal{T}^{*}}=0$ and therefore the steady state is given by

$$
\widehat{\mathbf{x}}=\left(\frac{1}{1+\rho_{o f f}^{m G} C_{2}}\left(C_{1}-C_{2}\right), 0,0,0, C_{2}\right)
$$

We now observe that the two parameter relations

$$
C_{2} \leq\left[m G A P_{t o t}\right] \quad \text { and } \quad C_{1} \geq C_{2}
$$

are sufficient for $[\widehat{t G E F}]=0$ and $\left[\widehat{t G E F^{*}}\right]=0$. In fact, if $C_{1} \geq C_{2}$ then by subtracting Eq. 3.32 from Eq. 3.31, we have

$$
\widehat{[m G]}+\widehat{\left[m G^{*}\right]}-[\widehat{t G E F}]=C_{1}-C_{2} \geq 0
$$

and hence $\widehat{[m G]}+\widehat{\left[m G^{*}\right]} \geq \widehat{[t G E F]}$. On the other hand, from Eq. 3.29, we must have $[\widehat{t G E F}]=0$ or $\widehat{\left[m G^{*}\right]}=0$. Thus if $\widehat{\left[m G^{*}\right]}=0$ then $\widehat{[m G]}=0$ (from Eq. 3.27) and hence the nonnegativeness implies $[\widehat{t G E F}]=0$. Hence we conclude that Eq. B.5 guarantee $[\widehat{t G E F}]=0$.

Now, Eq. 3.32 gives $\left[\widehat{t G E F}^{*}\right]=C_{2}-\left[\widehat{m G A P}^{*}\right]$ and from Eq. 3.30, we must have

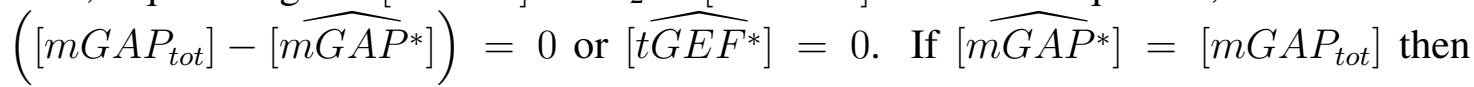
$\left[\widehat{t G E F}^{*}\right]=C_{2}-\left[m G A P_{t o t}\right] \leq 0$ (from Eq. B.5) and thus $\left[\widehat{t G E F}^{*}\right]=0$. Therefore, we have shown that Eq. B.5 implies $[\widehat{t G E F}]=0$ and $\left[\widehat{t G E F^{*}}\right]=0$. Consequently, the steady state in this case must be given by Eq. 3.36 . 
Local Stability Analysis. We begin reducing the ODE system with the conservation laws given by Eqs. 3.19 and 3.20. In fact, if we write

$$
[m G]=C_{1}-\left[m G^{*}\right]-\left[t G E F^{*}\right]-\left[m G A P^{*}\right] \quad \text { and } \quad[t G E F]=C_{2}-\left[t G E F^{*}\right]-\left[m G A P^{*}\right]
$$

then Eqs. 3.21 - 3.26 can be written in the form

$$
\begin{aligned}
\frac{d\left[m G^{*}\right]}{d t} & =f_{1}\left(\left[m G^{*}\right], \mathcal{T}^{*},\left[t G E F^{*}\right],\left[m G A P^{*}\right]\right) \\
\frac{d \mathcal{T}^{*}}{d t} & =f_{2}\left(\left[m G^{*}\right], \mathcal{T}^{*},\left[t G E F^{*}\right],\left[m G A P^{*}\right]\right) \\
\frac{d\left[t G E F^{*}\right]}{d t} & =f_{3}\left(\left[m G^{*}\right], \mathcal{T}^{*},\left[t G E F^{*}\right],\left[m G A P^{*}\right]\right) \\
\frac{d\left[m G A P^{*}\right]}{d t} & =f_{4}\left(\left[m G^{*}\right], \mathcal{T}^{*},\left[t G E F^{*}\right],\left[m G A P^{*}\right]\right)
\end{aligned}
$$

where

$$
\begin{aligned}
& f_{1}\left(\left[m G^{*}\right], \mathcal{T}^{*},\left[t G E F^{*}\right],\left[m G A P^{*}\right]\right)=\left(C_{1}-\left[m G^{*}\right]-\left[t G E F^{*}\right]-\left[m G A P^{*}\right]\right) \\
&-\rho_{o f f}^{t G}\left[m G A P^{*}\right]\left[m G^{*}\right]-\rho_{o n}^{I}\left(C_{2}-\left[t G E F^{*}\right]-\left[m G A P^{*}\right]\right)\left[m G^{*}\right], \\
& f_{2}\left(\left[m G^{*}\right], \mathcal{T}^{*},\left[t G E F^{*}\right],\left[m G A P^{*}\right]\right)=\rho_{o n}^{t G}\left[t G E F^{*}\right]\left(1-\mathcal{T}^{*}\right)-\rho_{o f f}^{t G} \mathcal{T}^{*}, \\
& f_{3}\left(\left[m G^{*}\right], \mathcal{T}^{*},\left[t G E F^{*}\right],\left[m G A P^{*}\right]\right)=\rho_{o n}^{I}\left(C_{2}-\left[t G E F^{*}\right]-\left[m G A P^{*}\right]\right)\left[m G^{*}\right] \\
&-\rho_{o n}^{I I}\left[t G E F^{*}\right]\left(\left[m G A P_{t o t}\right]-\left[m G A P^{*}\right]\right),
\end{aligned}
$$

and

$$
f_{4}\left(\left[m G^{*}\right], \mathcal{T}^{*},\left[t G E F^{*}\right],\left[m G A P^{*}\right]\right)=\rho_{o n}^{I I}\left[t G E F^{*}\right]\left(\left[m G A P_{t o t}\right]-\left[m G A P^{*}\right]\right)
$$

The eigenvalues of the Jacobian Matrix can be thus calculated for each one of the four steady states given by Eqs. 3.33 - 3.36. We prove that all steady states are LAS by showing that the eigenvalues of the Jacobian Matrix are all negative real numbers. We perform the calculations with MATLAB's R2019b symbolic toolbox and analyze each case separately (see supplementary file with MATLAB codes). We analyze each case separately.

(1) If $C_{1}>\left[m G A P_{t o t}\right]$ and $C_{2}>C_{1}$, the Jacobian matrix evaluated at the steady state given by Eq. 3.33 gives the eigenvalues

$$
\lambda_{1}=-\rho_{o n}^{m G}\left(C_{1}-\left[m G A P_{t o t}\right]\right) \quad \text { and } \quad \lambda_{2}=-\rho_{o n}^{t G}\left(C_{1}-\left[m G A P_{t o t}\right]\right)-\rho_{o f f}^{t G}
$$

which are negative. Moreover, the other eigenvalues $\lambda_{3}$ and $\lambda_{4}$ are such that 


$$
\lambda_{3}+\lambda_{4}=\rho_{o n}^{I}\left(C_{1}-C_{2}\right)-1-\rho_{o f f}^{m G}\left[m G A P_{t o t}\right]<0
$$

and

$$
\lambda_{3} \lambda_{4}=-\rho_{\text {on }}^{I}\left(C_{1}-C_{2}\right)>0
$$

and thus $\lambda_{3}$ and $\lambda_{4}$ are negative and hence the steady state is LAS.

(2) If $C_{2}>\left[m G A P_{t o t}\right]$ and $C_{1}>C_{2}$, the Jacobian matrix evaluated at the steady state given by Eq. 3.34 gives the eigenvalues

$$
\begin{aligned}
& \lambda_{1}=-\rho_{o f f}^{t G}-\rho_{o n}^{t G}\left(C_{2}-\left[m G A P_{t o t}\right]\right), \quad \lambda_{2}=-1-\rho_{o f f}^{m G}\left[m G A P_{t o t}\right], \\
& \lambda_{3}=-\rho_{o n}^{I I}\left(C_{2}-\left[m G A P_{t o t}\right]\right) \quad \text { and } \quad \lambda_{4}=-\frac{\rho_{o n}^{I}\left(C_{1}-C_{2}\right)}{\rho_{o f f}^{m G}\left[m G A P_{t o t}\right]+1}
\end{aligned}
$$

which are all negative and hence the steady state is LAS.

(3) If $C_{1}<\left[m G A P_{t o t}\right]$ and $C_{2}>C_{1}$, the Jacobian matrix evaluated at the steady state given by Eq. 3.35 gives the eigenvalues

$$
\lambda_{1}=\rho_{o n}^{I I}\left(C_{1}-\left[m G A P_{t o t}\right]\right) \quad \text { and } \quad \lambda_{2}=-\rho_{o f f}^{t G}
$$

which are negative. Moreover, the other eigenvalues $\lambda_{3}$ and $\lambda_{4}$ are such that

$$
\lambda_{3}+\lambda_{4}=\rho_{\text {on }}^{I}\left(C_{1}-C_{2}\right)-C_{1} \rho_{o f f}^{m G}-1<0
$$

and

$$
\lambda_{3} \lambda_{4}=-\rho_{\text {on }}^{I}\left(C_{1}-C_{2}\right)>0
$$

and thus $\lambda_{3}$ and $\lambda_{4}$ are negative and hence the steady state is LAS.

(4) If $C_{2}<\left[m G A P_{t o t}\right]$ and $C_{1}>C_{2}$, the Jacobian matrix evaluated at the steady state given by Eq. 3.36 gives the eigenvalues

$$
\lambda_{1}=-1-C_{2} \rho_{o f f}^{m G}, \quad \lambda_{2}=\rho_{o n}^{I I}\left(C_{2}-\left[m G A P_{t o t}\right]\right), \quad \lambda_{3}=-\rho_{o f f}^{t G}
$$

and 
bioRxiv preprint doi: https://doi.org/10.1101/2020.08.31.276311; this version posted December 15,2020 . The copyright holder for this preprint (which was not certified by peer review) is the author/funder, who has granted bioRxiv a license to display the preprint in perpetuity. It is made available under aCC-BY-ND 4.0 International license.

$$
\lambda_{4}=-\frac{\rho_{o n}^{I}\left(C_{1}-C_{2}\right)}{C_{2} \rho_{o f f}^{m G}+1}
$$

which are all negative and hence the steady state is LAS. 
Appendix C. Proof of Theorem 3.3

We proceed with the steady state analysis in the same way of Theorem 3.2. We consider the same four different cases and calculate the $\xi$-dependent families of steady states, where $\xi \geq 0$ represent the $\mathrm{tG}$ concentration. We also obtain necessary relationships for the conserved quantities $\tilde{C}_{2}, \tilde{C}_{1}$, and $\left[m G A P_{t o t}\right]$, as well as admissible intervals for $\xi$ that guarantee the existence of nonnegative steady states.

Case 1: $\widehat{\left[m G^{*}\right]}=0$ and $\left[{\widehat{m G A P^{*}}}^{*}=\left[m G A P_{t o t}\right]\right.$.

From Eq. 3.39, we have $\widehat{[m G]}=0$ and subtracting Eq. 3.38 from Eq. 3.37, we get $[\widehat{t G E F}]=\tilde{C}_{2}-\tilde{C}_{1} \geq 0$ only if $\tilde{C}_{2} \geq \tilde{C}_{1}$. Substituting $[\widehat{t G E F}]$ on the conservation law given by Eq. 3.38 and using Eq. 3.40 to write $\widehat{\left[t G^{*}\right]}=\frac{\rho_{o n}^{t G}\left[t \widehat{\left.G E F^{*}\right] \xi}\right.}{\rho_{o f f}^{t G}}$, we obtain

$$
\xi+\frac{\rho_{o n}^{t G}\left[\widehat{t G E F^{*}}\right] \xi}{\rho_{o f f}^{t G}}+\left(\tilde{C}_{2}-\tilde{C}_{1}\right)+\left[\widehat{t G E F^{*}}\right]+\left[m G A P_{t o t}\right]=\tilde{C}_{2}
$$

and hence

$$
\left[{\widehat{t G E F^{*}}}^{*}=\left(\tilde{C}_{1}-\left[m G A P_{t o t}\right]-\xi\right]\right) \frac{\rho_{o f f}^{t G}}{\rho_{o f f}^{t G}+\rho_{o n}^{t G \xi}}
$$

only if $\tilde{C}_{1}-\left[m G A P_{t o t}\right] \geq \xi$. Therefore, in this case the $\xi$-dependent family of steady states is given by

$$
\begin{aligned}
\widehat{\mathbf{x}}_{\xi}= & \left(0,0, \xi, \frac{\left(\tilde{C}_{1}-\left[m G A P_{t o t}\right]-\xi\right) \rho_{o n}^{t G} \xi}{\rho_{o f f}^{t G}+\rho_{o n}^{t G} \xi}, \tilde{C}_{2}-\tilde{C}_{1}\right. \\
& \left.\left(\tilde{C}_{1}-\left[m G A P_{t o t}\right]-\xi\right) \frac{\rho_{o f f}^{t G}}{\rho_{o f f}^{t G}+\rho_{o n}^{t G} \xi},\left[m G A P_{t o t}\right]\right)
\end{aligned}
$$

Case 2: $[\widehat{t G E F}]=0$ and $[\widehat{m G A P} *]=\left[m G A P_{t o t}\right]$

Using Eq. 3.39 to write $\widehat{[m G]}=\rho_{o f f}^{m G}\left[m G A P_{t o t}\right] \widehat{\left[m G^{*}\right]}$ and subtracting Eq. 3.38 from Eq. 3.37, we obtain the expressions for $\left[m G^{*}\right]$ and $[m G]$

$$
\widehat{\left[m G^{*}\right]}=\frac{\left(\tilde{C}_{1}-\tilde{C}_{2}\right)}{\rho_{o f f}^{t G}\left[m G A P_{t o t}\right]+1} \quad \text { and } \quad \widehat{[m G]}=\frac{\left(\tilde{C}_{1}-\tilde{C}_{2}\right) \rho_{o f f}^{t G}\left[m G A P_{t o t}\right]}{\rho_{o f f}^{t G}\left[m G A P_{t o t}\right]+1}
$$

and thus we must have $\tilde{C}_{1} \geq \tilde{C}_{2}$. Now looking at Eq. 3.38 and substituting $\quad \widehat{\left[t G^{*}\right]}=$ $\frac{\rho_{\text {on }}^{t G}\left[t \widehat{G E F}^{*}\right] \xi}{\rho_{\text {off }}^{t G}}$ from Eq. 3.40, we obtain 


$$
\left.\left[\widehat{t G E F^{*}}\right]=\left(\tilde{C}_{2}-\left[m G A P_{t o t}\right]-\xi\right]\right) \frac{\rho_{o f f}^{t G}}{\rho_{o f f}^{t G}+\rho_{o n}^{t G \xi}}
$$

only if $\tilde{C}_{2}-\left[m G A P_{t o t}\right] \geq \xi$. Therefore, in this case the $\xi$-dependent family of steady states is given by

$$
\begin{aligned}
\widehat{\mathbf{x}}_{\xi}= & \left(\frac{\left(\tilde{C}_{1}-\tilde{C}_{2}\right) \rho_{o f f}^{m G}\left[m G A P_{t o t}\right]}{1+\rho_{o f f}^{m G}\left[m G A P_{t o t}\right]}, \frac{\left(\tilde{C}_{1}-\tilde{C}_{2}\right)}{1+\rho_{o f f}^{m G}\left[m G A P_{t o t}\right]},\right. \\
& \left.\xi, \frac{\left.\left(\tilde{C}_{2}-\left[m G A P_{t o t}\right]-\xi\right]\right) \rho_{o n}^{t G} \xi}{\rho_{o f f}^{t G}+\rho_{o n}^{t G \xi} \xi}, 0, \frac{\left.\left(\tilde{C}_{2}-\left[m G A P_{t o t}\right]-\xi\right]\right) \rho_{o f f}^{t G}}{\rho_{o f f}^{t G}+\rho_{o n}^{t G \xi} \xi},\left[m G A P_{t o t}\right]\right)
\end{aligned}
$$

Case 3: $\widehat{\left[m G^{*}\right]}=0$ and $\left[\widehat{t G E F^{*}}\right]=0$

From Eqs. 3.39 and 3.40, we have $\widehat{[m G]}=0$ and $\widehat{\left[t G^{*}\right]}=0$, respectively. Subtracting Eq. 3.38 from Eq. 3.37, in this case we get $[\widehat{t G E F}]=\tilde{C}_{2}-\tilde{C}_{1} \geq 0$ only if $\tilde{C}_{2} \geq \tilde{C}_{1}$. Now, from the conservation law given by Eq. 3.37, we obtain $\left[\widehat{m G A P}^{*}\right]=\tilde{C}_{1}-\xi$ and thus $\left[\widehat{m G A P}^{*}\right] \in\left[0,\left[m G A P_{t o t}\right]\right]$ only if $\max \left(0, \tilde{C}_{1}-\left[m G A P_{t o t}\right]\right) \leq \xi \leq \tilde{C}_{1}$. In this case, the $\xi$-dependent family of steady states is given by

$$
\widehat{\mathbf{x}}_{\xi}=\left(0,0, \xi, 0, \tilde{C}_{2}-\tilde{C}_{1}, 0, \tilde{C}_{1}-\xi\right) .
$$

Case $4:[\widehat{t G E F}]=0$ and $\left[\widehat{t G E F} \widehat{t}^{*}\right]=0$

Eq. 3.40 gives $\widehat{\left[t G^{*}\right]}=0$ and the conservation law given by Eq. 3.38 yields $\left[\widehat{m G A P}^{*}\right]=$ $\tilde{C}_{2}-\xi$. Now using Eq. 3.39 to write $\widehat{[m G]}=\rho_{o f f}^{m G}\left(\tilde{C}_{2}-\xi\right) \widehat{\left[m G^{*}\right]}$, the conservation law given by Eq. 3.38 gives

$$
\widehat{\left[m G^{*}\right]}=\frac{\left(\tilde{C}_{1}-\tilde{C}_{2}\right)}{1+\rho_{o f f}^{m G}\left(\tilde{C}_{2}-\xi\right)} \quad \text { and } \widehat{[m G]}=\frac{\rho_{o f f}^{m G}\left(\tilde{C}_{1}-\tilde{C}_{2}\right)\left(\tilde{C}_{2}-\xi\right)}{1+\rho_{o f f}^{m G}\left(\tilde{C}_{2}-\xi\right)}
$$

and since $\left[m G A P^{*}\right] \in\left[0,\left[m G A P_{t o t}\right]\right]$ and the steady states must be nonnegative, we must have

$$
\max \left(0, \tilde{C}_{2}-\left[m G A P_{t o t}\right]\right) \leq \xi \leq \tilde{C}_{2} \leq \tilde{C}_{1}
$$

The $\xi$-dependent familiy of steady states is therefore given by

$$
\widehat{\mathbf{x}}_{\xi}=\left(\frac{\rho_{o f f}^{m G}\left(\tilde{C}_{1}-\tilde{C}_{2}\right)\left(\tilde{C}_{2}-\xi\right)}{1+\rho_{o f f}^{m G}\left(\tilde{C}_{2}-\xi\right)}, \frac{\left(\tilde{C}_{1}-\tilde{C}_{2}\right)}{1+\rho_{o f f}^{m G}\left(\tilde{C}_{2}-\xi\right)}, \xi, 0,0,0, \tilde{C}_{2}-\xi\right)
$$

